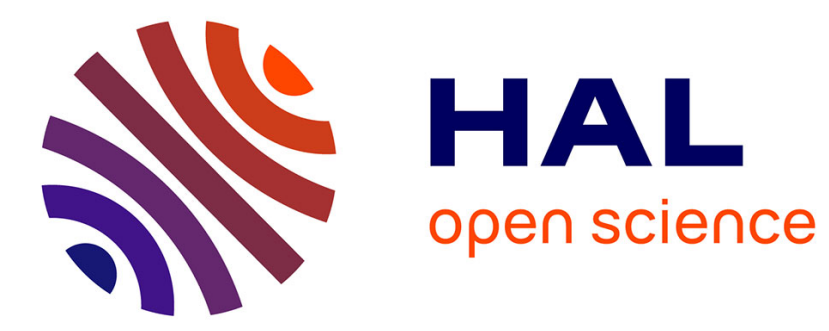

\title{
Forçages météo-marins et dynamique morphosédimentaire saisonnière des cordons dunaires. Exemple de la baie de Saint-Michel-en-Grève (Côtes d'Armor, Bretagne)
}

Serge S. Suanez, Pierre Stéphan

\section{To cite this version:}

Serge S. Suanez, Pierre Stéphan. Forçages météo-marins et dynamique morphosédimentaire saisonnière des cordons dunaires. Exemple de la baie de Saint-Michel-en-Grève (Côtes d'Armor, Bretagne). Géomorphologie: relief, processus, environnement, 2006, 12 (2), pp.91-110. 10.4000/geomorphologie.529 . hal-00270021

\section{HAL Id: hal-00270021 \\ https://hal.science/hal-00270021}

Submitted on 3 Apr 2008

HAL is a multi-disciplinary open access archive for the deposit and dissemination of scientific research documents, whether they are published or not. The documents may come from teaching and research institutions in France or abroad, or from public or private research centers.
L'archive ouverte pluridisciplinaire HAL, est destinée au dépôt et à la diffusion de documents scientifiques de niveau recherche, publiés ou non, émanant des établissements d'enseignement et de recherche français ou étrangers, des laboratoires publics ou privés. 


\title{
Forçages météo-marins et dynamique morphosédimentaire saisonnière des cordons dunaires. Exemple de la baie de Saint-Michel-en-Grève (Côtes d'Armor, Bretagne)
}

\section{Meteo-marine forcings and seasonal morphosedimentary dynamic of dunes. Example of the bay of Saint-Michel- en-Grève (Côtes d'Armor, Bretagne)}

\author{
Serge Suanez* et Pierre Stéphan*
}

\begin{abstract}
Résumé
La présente étude se propose d'examiner le rôle des forçages météorologiques et marins dans la dynamique des cordons dunaires à partir de données acquises en baie de Saint-Michel-en-Grève. Elle repose sur un suivi topo-morphologique à haute fréquence des cordons dunaires du Grand Rocher et de Saint-Michel-en-Grève réalisé entre les mois d'octobre 2002 et de septembre 2003. Deux types de relevés de terrain ont été utilisés afin de cerner au mieux les modifications morphosédimentaires des dunes : la superficie et la morphologie dunaire le long de six profils. À cela s'ajoute un travail d'analyse des conditions météo-marines enregistrées durant la période d'observation (houle, marée, vent et pression barométrique). À partir de ces données, l'Analyse statistique en Composante Principale (ACP) suggère que les niveaux extrêmes pourraient jouer un rôle important dans l'érosion des cordons dunaires, représentés essentiellement par les phénomènes de run-up associés aux fortes houles. Pour autant, les périodes de faible activité morphogène permettent une régénération non négligeable de la dune, gommant ainsi l'impact morphosédimentaire immédiat des tempêtes. Ces mécanismes brouillent quelque peu le schéma du fonctionnement saisonnier.
\end{abstract}

Mots clés : cordon dunaire, forçage météo-marin, suivi topo-morphologique, Analyse en Composante Principale, niveau extrême, érosion.

\begin{abstract}
This research concerns the role played by meteorological and marine forcings on coastal dune dynamics by analysing a data set collected in the bay of Saint-Michel-en-Grève. The study is based on a high frequency monitoring of the dunes of Grand Rocher and Saint-Michel-en-Grève realised between october 2002 and september 2003. Two types of field measurements were used to analyse the morphosedimentary changes of the dune: surface changes and dune morphology along six transects. In addition, an analysis of meteorological and oceanographic conditions during the survey period was carried out using wave, tide, wind, and atmospheric pressure data. The synthesis of these data using the statistical Principal Component Analysis shows the important role played by high water levels on dunes erosion, mainly in response to run-up processes related to strong waves. Nevertheless, periods characterised by low energetic conditions allow progressive dune regeneration which limits the morphosedimentary impacts of storms events. These mechanisms make more complex and irregular the seasonal morphological change pattern.
\end{abstract}

Key words: coastal dune, meteo-marine forcing agent, monitoring, Principal Component Analysis, high water level, erosion.

\section{Abridged English Version}

Between October 2002 and September 2003 high frequency monitoring of the coastal dunes of Grand Rocher and Saint-Michel-en-Grève located on the Saint-Michel-enGrève bay were undertaken. This work was planned by the local authority (Conseil général des Côtes d'Armor) because of recent erosion, which affected these dunes. The aims of this study are to present results obtained during this survey and to analyse the role played by meteorological and marine forcings in dune morphological changes observed during this period.

* GÉOMER, UMR 6554 CNRS, Institut Universitaire Européen de la Mer, Place Nicolas Copernic, 29280 Plouzané. Courriel : serge.suanez@univbrest.fr ; pierre.stephan@univ-brest.fr 
The studied area of Saint-Michel-en-Grève bay is located on the northern part of Brittany, in the county of Côtes d'Armor (fig. 1). It is characterised by small foredunes distributed in three places: dunes of Saint-Efflam on the western part of the bay, dunes of Saint-Michel-en-Grève on the eastern part and dunes of Grand Rocher in the centre (fig. 1). The survey was carried out on the two last sites, dunes of Grand Rocher and Saint-Michel-en-Grève. They are both characterised by a flat morphology and their height does not exceed $1.5 \mathrm{~m}$. Nevertheless, the foredune of Saint-Michel-en-Grève is more developed than the one of Grand Rocher in terms of surface and length (figs. 2 and 3). Morphosedimentay dynamics are strongly driven by wind blowing principally from the west to the south-west during the autumnal and winter period. These winds are related to the Atlantic disturbance reaching the western part of Europe. Therefore, they induce erosional processes during these periods through the generation of waves. North-east wind is also important. It blows essentially during the spring period inducing accretional processes probably related to lower-energy constructional waves (fig. 4).

The monitoring was carried out using laser tacheometer instruments. Two kinds of measurements were realised. The first one concerned measurement of the front of the dune in order to quantify dune surface changes. The second was realised along six dunes transects (figs. 2 and 3) to analyse and quantify cross-shore morphology changes. The results showed an important loss of sediment for Grand Rocher foredune reaching $332 \pm 5 \mathrm{~m}^{2}$ during the survey period (fig. 5). The erosion principally occurred during the winter period; conversely spring and summer periods were characterised by accretion. On the contrary, Saint-Michel-enGrève coastal dunes were affected by significant accretion. Surface gained during the survey reached $3363 \pm 28 \mathrm{~m}^{2}$ (fig. 6). The accretional process was constant for the whole period. Such evolution is also observed when looking at the beach/dune changes along profile measurements (figs. 7 and 8). Profiles 1 and 2 located on the Grand Rocher foredune showed that the erosion mainly concerned the western part of the dune which lost $-4 \mathrm{~m}^{3} / l . m$ over the whole period (fig. 7). Profiles 3, 4, 5 and 6 located on Saint-Michel-enGrève foredune were characterised by sediment accumulation reaching +1 to $+2.5 \mathrm{~m}^{3} / \mathrm{l} . \mathrm{m}$ (fig. 8).

Driving agents such as wave, tide wind, and atmospheric pressure were analysed to identify storm events and periods of calm (fig. 9). Wave data were obtained from a Météo France, CETMEF and LNHE-EDF wave data base for the Atlantic, Channel and North Sea coasts based on TOMAWAC running model. Tide gauge records were obtained from the Service Hydrographique et Océanographique de la Marine (SHOM). Calculation of run-up was undertaken using P. Ruggiero (2001) formula:

$$
R^{T}{ }_{2 \%}=0.27 \sqrt{S H_{o} L_{o}}
$$

where $S$ : beach slope $(\tan \beta) ; H_{o}$ : wave height $\left(H_{m o}\right) ; L_{o}$ : wave length $=g T^{2} / 2 \pi=1,561 T^{2}\left(g:\right.$ gravity $=9.81 \mathrm{~m} / \mathrm{s}^{2}$; $T=T_{\text {pic }}:$ wave period).
Based on this data set, it appeared that the period running from October 2002 to May 2003 was characterised by 13 high energy geomorphic events (tab. 3). Storms characterised by high amplitude waves $(>4 \mathrm{~m}$ ) and/or high water level $(>4 \mathrm{~m})$ were identified as significant events in terms of geomorphic effects. This energetic period was followed by a long period of calm from May 2003 to September 2003 (fig. 9). In order to analyse the role played by forcing agents in dune morphosedimentary changes, statistical analysis using Principal Component Analysis (PCA) was achieved (fig. 10). Results showed the important role played by high water levels mainly induced by run-up processes associated to strong wave (figs. 11 and 12). High tide water level represents a minor factor when it is not combined with a storm event.

Potential aeolian sand transport was calculated using R.A. Bagnolds (1941) and R. Kawamura (1964) for each period interval corresponding to the measurements (tab. 5): (Bagnolds, 1941) $Q=C(d / D)^{0,5} V^{*}{ }_{c}^{3}(\rho a / g)$ (Kawamura, 1964) $Q=K(\rho a / g)\left(V^{*}-V^{*}{ }_{c}\right)\left(V^{*}+V^{*}{ }_{c}\right)^{2}$ where $Q$ : sand transport $(\mathrm{g} / \mathrm{cm} / \mathrm{s}) ; V^{*}:$ wind speed $\mathrm{cm} / \mathrm{s}$; $V_{c}^{*}$ : threshold wind velocity $\mathrm{cm} / \mathrm{s} ; C$ : constant depending on sediment sorting $=1.5$ very well sorted, 1.8 well sorted, 2.8 poarly sorted, 3.5 very poarly sorted; $K$ : constant of 2.7 for grain sand of 100 to $800 \mu \mathrm{m}$; $\rho$ a: air density $\left(1.23 \mathrm{~kg} / \mathrm{m}^{3}\right)$; g: gravity $\left(9.81 \mathrm{~m} / \mathrm{s}^{2}\right)$; d: grain diameter $(\mathrm{cm})$; D: standard grain diameter $(0.025 \mathrm{~cm})$.

This approach allowed us to show that the morphosedimentary impacts of storm events where limited when aeolian sand transport was important, mainly during dry periods. These short phases of dune accumulation make more complex the seasonal behaviour of the dune system, which is characterised by erosional processes during winter and dune regeneration during summer, especially when a dry period is immediately following a storm event.

\section{Introduction}

À la demande du Conseil général des Côtes d'Armor, un suivi topo-morphologique à haute fréquence des deux cordons dunaires du Grand Rocher et de Saint-Michel-enGrève, situés en baie de Saint-Michel-en-Grève (fig. 1), a été entrepris entre les mois d'octobre 2002 et septembre 2003. Ce travail fut décidé alors que des phénomènes d'érosion affectaient ces derniers (Suanez, 2004 ; Stéphan et Suanez, 2004), afin d'analyser et de comprendre les mécanismes à l'origine de leur recul.

Cet article présente les résultats obtenus durant cette année de suivi topo-morphologique et examine le rôle des forçages météorologiques dans l'évolution des cordons dunaires étudiés. Les travaux réalisés sur le fonctionnement saisonnier des milieux dunaires insistent sur la complexité des processus alternant entre phase d'érosion et phase d'alimentation (Davidson-Arnott et Law, 1990 ; Pye, 1991 ; Sherman et Bauer, 1993). Comme de nombreux auteurs l'ont montré, les tempêtes, épisodes morphogènes générant une élévation brutale et temporaire du niveau marin jouent un rôle important dans l'érosion des dunes (Sherman et Nordstrom, 1985 ; 
Fig. 1 - Carte de localisation.

Fig. 1 - Location map.
Carter et al., 1990 ; Pye et Neal, 1994 ; Arens, 1996a). Ces phénomènes érosifs sont renforcés lorsqu'une onde de tempête se combine à une pleine mer de viveeau (Ruz et Meur-Ferrec, 2004 ; Battiau-Queney, 2004). Toutefois, comme le rappelle P.A. Pirazzoli (2000), aucune situation combinant une très forte tempête et une marée de vive-eau ne s'est encore produite sur le littoral nord atlantique, à l'exception peut-

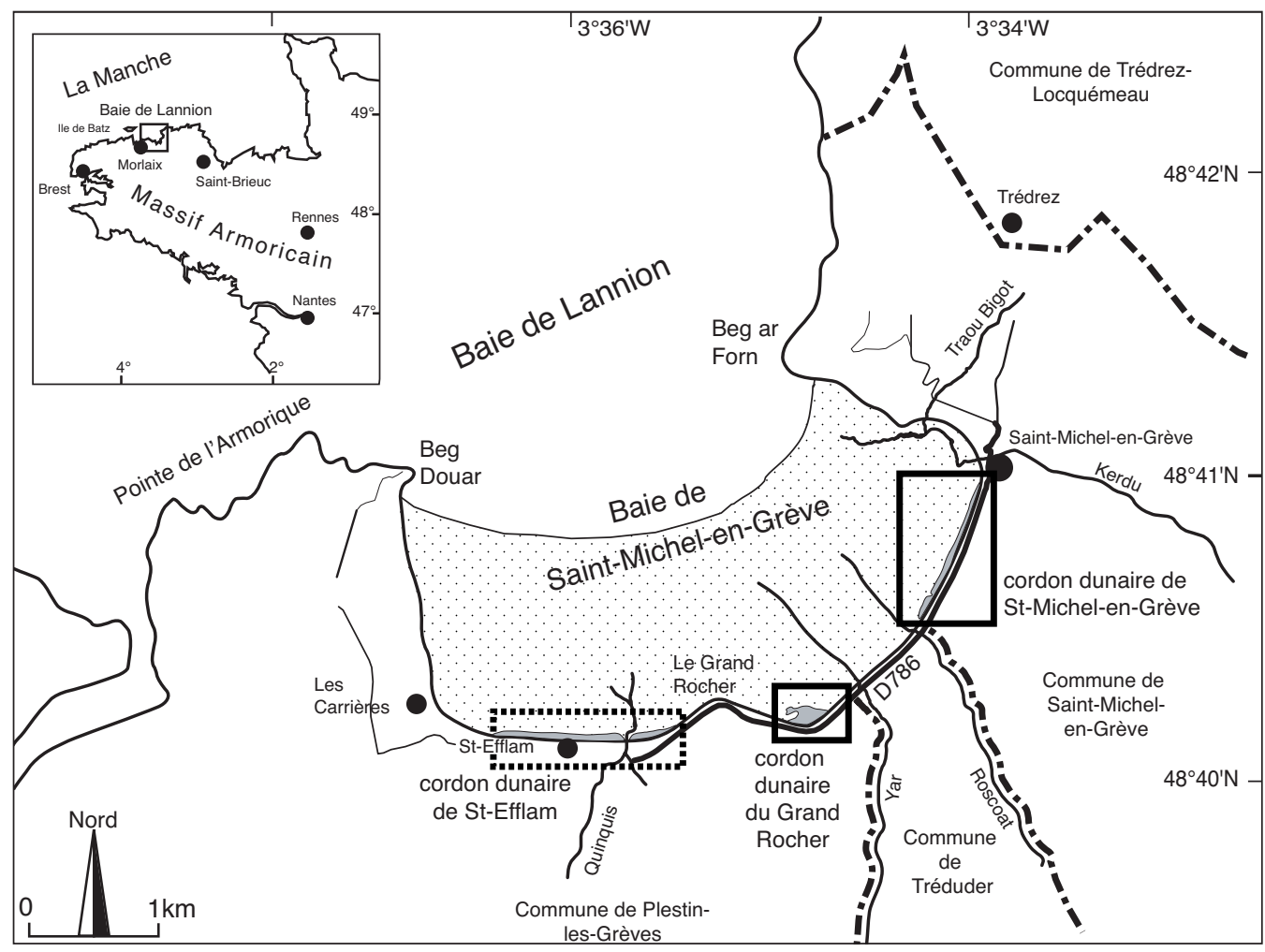
être de la tempête de l'hiver 1989-1990 qui a eu sur le littoral breton des effets spectaculaires et dévastateurs (Fichaut et Hallégouët, 1989). Les travaux réalisés sur les relations entre les niveaux marins extrêmes et l'érosion des dunes apportent un canevas méthodologique intéressant (Ruggiero et al., 1997 ; Dail et al., 2000 ; Ruggiero et al., 2001). Ces auteurs soulignent le rôle important des fortes houles et des phénomènes de run-up qui leur sont associés dans la dynamique de recul des fronts de dune. Pour autant, cette érosion n'est pas totalement irréversible car des phases d'alimentation et de régénération des cordons dunaires peuvent prendre place en période estivale où l'activité morphogène est considérablement réduite (Psuty, 1988 ; Davidson-Arnott et Law, 1990 ; Arens, 1996a, 1997 ; Ruz et Meur-Ferrec, 2004). La réversibilité des effets de l'érosion peut également s'inscrire dans un temps beaucoup plus long, pluriannuel, comme l'ont montré des suivis réalisés sur diverses plages (Thom et Hall, 1991 ; Battiau-Queney et al., 2000). Aussi, une bonne connaissance de ces fonctionnements est une aide indispensable à la gestion rationnelle de ces milieux (Hesp, 2002 ; Ruz, 2004 ; Battiau-Queney, 2004).

D'un point de vue méthodologique, deux types de levés ont été réalisés afin de mesurer la variabilité saisonnière du bilan sédimentaire des cordons dunaires étudiés. Le premier repose sur la mesure de la superficie dunaire donnant ainsi une vision globale de l'évolution en plan des cordons. Le second s'est attaché à mesurer les changements morphosédimentaires du système cordon dunaire / haut de plage le long de six transects perpendiculaires au trait de côte permettant ainsi d'analyser le comportement du front de dune dans sa dimension verticale. L'étude des conditions de forçages météo-marins a été réalisée à partir de données Météo France pour le vent et la pression barométrique, d'enregis- trements marégraphiques fournies par le SHOM (Service Hydrographique et Océanographique de la Marine-Brest) et de données de houle issues d'une modélisation acquises auprès du LNHE-EDF (Laboratoire National d'Hydraulique et Environnement de l'EDF - Chatou). Dans un dernier point, l'analyse des données morphosédimentaires et des conditions de forçages météo-marins s'est faite à partir d'une série d'Analyses factorielles en Composantes Principales (ACP).

\section{Présentation de la zone d'étude}

La baie de Saint-Michel-en-Grève correspond à une plage de fond de baie cernée par de hautes falaises dont l'altitude décroît d'ouest en est de quatre-vingts mètres à une cinquantaine de mètres. Elle est ouverte aux flux océaniques d'ouest à nord-ouest et il n'est pas rare d'avoir des régimes de nord-est, principalement au printemps. Toutefois, la présence des pointes de Berg ar Forn à l'est et de Beg Douar à l'ouest confère à la baie un caractère abrité limitant les impacts morphogènes. L'importance du marnage (9,3 m à Locquirec) explique la grande superficie de l'estran $\left(7 \mathrm{~km}^{2}\right)$ qui peut découvrir sur plus de deux kilomètres de distance. Les cordons dunaires occupent un espace extrêmement réduit (25 $000 \mathrm{~m}^{2}$ environ, soit $0,04 \%$ du système littoral). Cela s'explique en grande partie par le «blocage » du système littoral par les falaises de fond de baie qui n'a pas permis l'édification d'une zone supralittorale étendue où s'exercerait la dynamique éolienne. À cela s'ajoute également le rôle des aménagements avec la construction de la route littorale D786. Cette dernière a repoussé « artificiellement » le trait de côte vers l'aval du système entraînant ainsi une submersion plus fréquente du haut d'estran et, avec elle, la destruc- 
tion des premières dunes (Gad et al., 2003). Les seules dunes holocènes que l'on puisse trouver en baie de SaintMichel-en-Grève se situent dans les rentrants que forment les débouchés des vallées fluviales. Elles sont aujourd'hui totalement masquées par des constructions d'origine anthropique que l'on a édifiées sur leurs revers (habitats, activités touristiques balnéaires, etc.).

La baie de Saint-Michel-en-Grève connaît un engraissement très important que l'on mesure particulièrement bien depuis le début du XIX ${ }^{\mathrm{e}}$ siècle (Suanez et al., 2002 ; Stéphan et Suanez, 2005). Depuis 1990, ces apports sédimentaires sableux ont entraîné un exhaussement suffisamment conséquent du haut de plage pour que se mettent en place des dunes bordières (Stéphan et Suanez, 2004). Elles se répartissent en trois cordons dunaires bien distincts. Les deux premiers, les cordons dunaires de Saint-Efflam et du Grand Rocher, se situent sur la commune de Plestin-les-Grèves, le troisième, de Saint-Michel-en-Grève, est situé à l'est-nordest sur la commune du même nom (fig. 1). D'un point de vue morphologique, ces « micro-reliefs dunaires» correspondent à des placages éoliens très peu élevés dont la partie externe forme un bourrelet inférieur à un mètre d'altitude par rapport au niveau des plus hautes mers. La granulométrie de ces derniers est très homogène d'un secteur à l'autre de la baie. Les mesures effectuées sur l'ensemble des sables dunaires montrent des variations très faibles comprises entre $163 \mu \mathrm{m}$ et $142 \mu \mathrm{m}$, pour un grain moyen égal à $155 \mu \mathrm{m}$. C'est en général un sédiment bien trié comme l'indiquent les indices de tri égal à 0,41 et d'asymétrie proche de 0 (Stéphan, 2003). Notre attention portera sur les deux cordons dunaires du Grand Rocher et de Saint-Michel-en-Grève qui font l'objet de cet article.

Le cordon dunaire du Grand Rocher occupe la partie centrale de la baie. Sa superficie ne dépasse pas $1900 \mathrm{~m}^{2}$ et sa hauteur reste inférieure à $1,5 \mathrm{~m}$. Il présente une morphologie bosselée constituée de bourrelets peu prononcés parallèles au rivage et séparés les uns des autres par des secteurs plans de type «plate-forme » (fig. 2). Son orientation et sa localisation font qu'il est protégé des flux d'ouest par le promontoire du Grand Rocher. À l'inverse, il est particulièrement bien exposé aux flux d'est à nord-est. Il apparaît extrêmement végétalisé (taux de couverture végétale > $90 \%$ ). Les formations végétales qui le couvrent sont des plus communes : dans la partie externe les graminées prédominent (Agropyrum junceinforme et Carex arenaria), la partie interne, proche de l'enrochement, est quant à elle couverte d'associations de pelouse rase (plantin, euphorbe, fucus et des lichens).

Le cordon dunaire de Saint-Michel-en-Grève est de taille plus importante ; il s'étend sur $800 \mathrm{~m}$ et couvre environ $14500 \mathrm{~m}^{2}$. Sa hauteur reste inférieure à 1,5 m. La morphologie de cet ensemble est similaire à celle décrite pour le cordon dunaire du Grand Rocher (fig. 3). À l'inverse, son orientation ouest-nord-ouest l'expose principalement aux flux océaniques d'ouest rentrant dans la baie. Son taux de couverture végétale est également très important (proche de $100 \%$ ) et présente les mêmes associations vétégales que celles décrites précédemment.
La dynamique éolienne est en grande partie conditionnée par les flux météo-marins d'ouest. Néanmoins, suivant les périodes de l'année, les vents de secteur est à nord-est jouent un rôle non négligeable. Les données de vent obtenues auprès de la station météorologique de Lannion permettent de décrire une « climatologie » générale et saisonnière des vents dans le secteur de la baie de Saint-Michel-en-Grève (fig. 4).

À l'échelle annuelle, la majorité des vents les plus forts est de direction ouest à sud-ouest $\left(220^{\circ}\right.$ à $\left.260^{\circ}\right)$. Ces vents accompagnent le passage des perturbations atlantiques génératrices de tempêtes sur le littoral. Les circulations d'est à nord-est $\left(40^{\circ}\right.$ à $\left.100^{\circ}\right)$ représentent la deuxième principale direction des vents annuels. Ils montrent toutefois des vitesses moins importantes que pour les vents d'ouest. À l'échelle saisonnière, une variation non négligeable des directions et des forces de vents est observée. Durant l'hiver, du mois de janvier au mois de mars, les vents de sud-ouest $\left(200^{\circ}\right.$ à $\left.260^{\circ}\right)$ sont les plus fréquents et les plus virulents (13\% du régime total compris entre 4 et $8 \mathrm{~m} / \mathrm{s}$ et $8 \%>$ à $8 \mathrm{~m} / \mathrm{s}$ ). Ces vents forts sont souvent associés à des hauts niveaux marins où peuvent se combiner marée de vive-eau et onde de tempête (surcote). Ces conditions sont propices à l'érosion des dunes. À partir du mois d'avril, les vents d'ouest sont moins intenses. Ils prennent une direction ouest-nord-ouest $\left(260^{\circ}\right.$ à $\left.300^{\circ}\right)$. Dans le même temps, on observe une nette recrudescence des vents d'est à nord-est $\left(40^{\circ}\right.$ à $\left.80^{\circ}\right)$ toutefois peu vigoureux (majorité du régime < à $4 \mathrm{~m} / \mathrm{s}$ ). Cette période printanière associant des vents morphogènes et une diminution des pluies est plutôt favorable à l'engraissement des dunes, aussi bien de Saint-Michel-enGrève que du Grand Rocher. En été, du mois de juillet au mois de septembre, les vents faiblissent quelle que soit la direction. Durant cette période « sèche », les vents les plus morphogènes soufflent de l'ouest. Cette circulation est alors propice à l'alimentation des dunes de Saint-Michelen-Grève. À partir du mois d'octobre, les perturbations atlantiques reviennent et s'accompagnent d'une augmentation de la force des vents de sud-ouest $\left(200^{\circ}\right.$ à $\left.260^{\circ}\right)$. Ces flux océaniques sont les plus représentés durant la période automnale. Ils s'accompagnent de hauts niveaux marins (marée d'équinoxe et ondes de tempêtes) capables de créer de graves dommages sur les cordons dunaires.

\section{Évolution morphosédimentaire des cordons dunaires du Grand Rocher et de Saint-Michel-en-Grève}

\section{Méthode et acquisition des données}

Entre les mois d'octobre 2002 et septembre 2003, nous avons réalisé onze campagnes de mesures topo-morphologiques des cordons dunaires du Grand Rocher et de Saint-Michel-en-Grève (tab. 1). La fréquence des relevés a été particulièrement élevée durant la période hivernale afin de cerner tous les épisodes de forte activité morphogène. À partir du printemps, lorsque les conditions anticycloniques ont prédominé, la fréquence est passée à un relevé par 


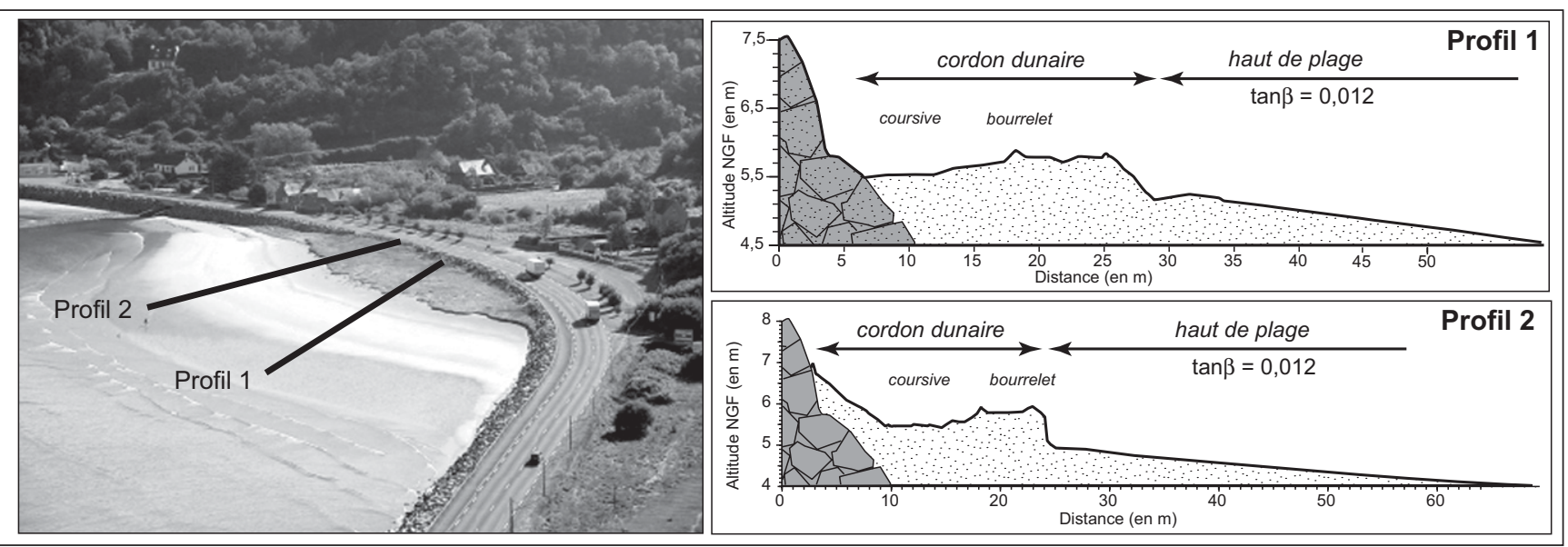

Fig. 2 - Morphologie du cordon dunaire et du haut de plage du Grand Rocher (cliché : Serge Suanez).

Fig. 2 - Morphology of the coastal dune and upper beach of Grand Rocher (photo: Serge Suanez).

mois. Ces levés de terrain ont été effectués au tachéomètre laser (Nicon DTM 300 et Leica TCR303). Ils sont composés d'un levé du front de dune afin de mesurer le recul ou l'avancée de ce dernier et de mesurer les surfaces dunaires perdues ou gagnées. La limite retenue pour délimiter le front de dune est clairement matérialisée par une rupture de pente marquant le contact entre la dune et le haut de plage. Cet escarpement dessine également la limite de la végétation dunaire. L'analyse quantitative de ces relevés (calcul des surfaces) a été réalisé sous SIG (ArcView 8.3) suivant une procédure classique d'intégration des données. Les levés de terrain sont aussi composés de mesures effectuées le long de six profils transversaux disposés perpendiculairement au trait de côte englobant la dune et le haut de plage : quatre profils sur le cordon dunaire de Saint-Michel-en-Grève (fig. 3) et deux sur celui du Grand Rocher (fig. 2). À partir de ces données, il a été possible de quantifier les volumes de sable perdus ou gagnés par la dune en utilisant la méthode des «surfaces verticales ». La superposition de deux profils délimite des aires en accrétion et en érosion entre chaque nœud d'intersection. Le calcul de la «surface verticale » revient à mesurer la superficie de ces aires par un calcul d'intégrale. En ramenant ces superficies au mètre linéaire, on obtient des

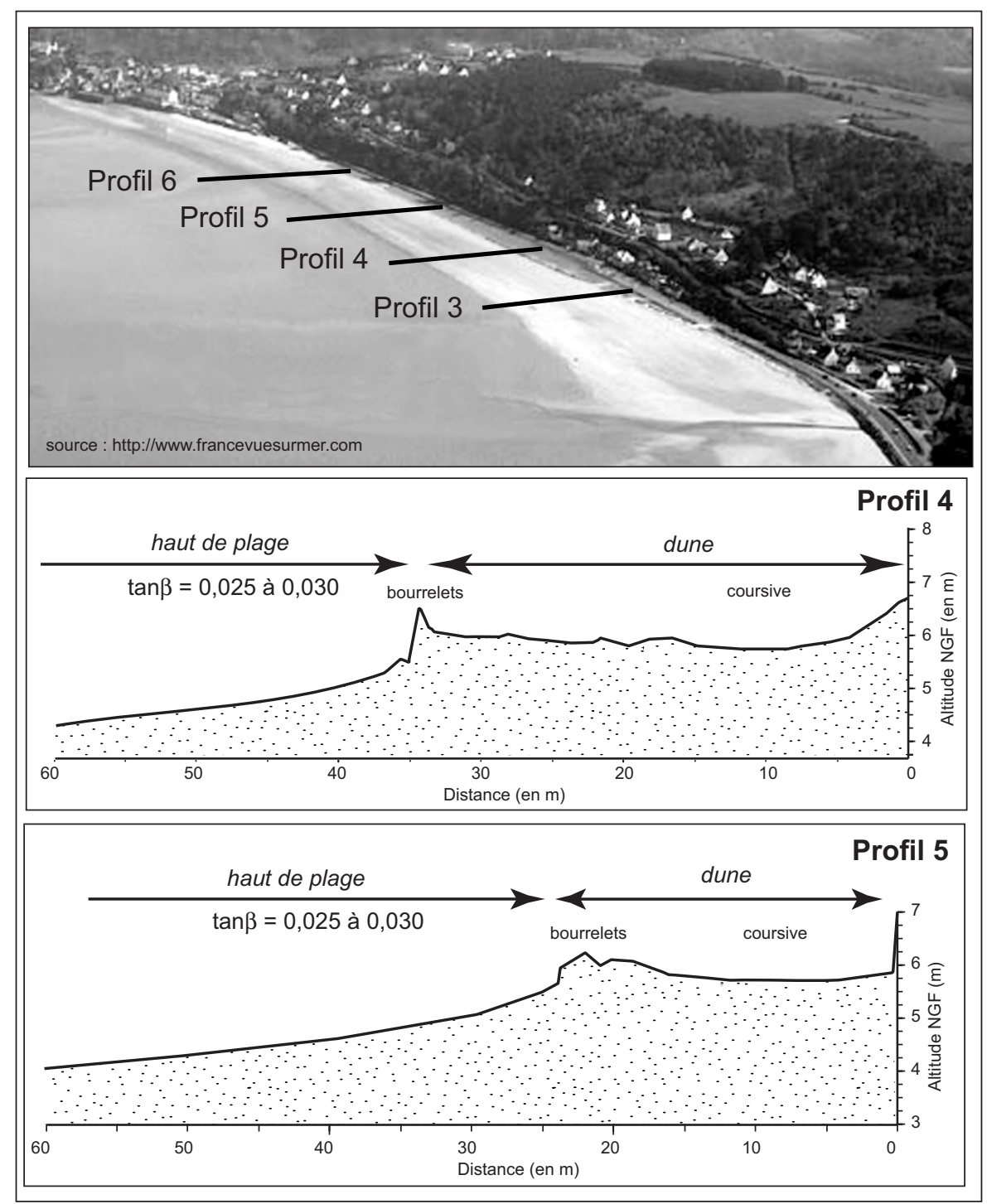

Fig. 3 - Morphologie du cordon dunaire et du haut de plage de Saint-Michel-en-Grève (source : http:/www.francevuesurmer.com).

Fig. 3 - Morphology of the coastal dune and upper beach of Saint-Michel-en-Grève (http:/www.francevuesurmer.com). 


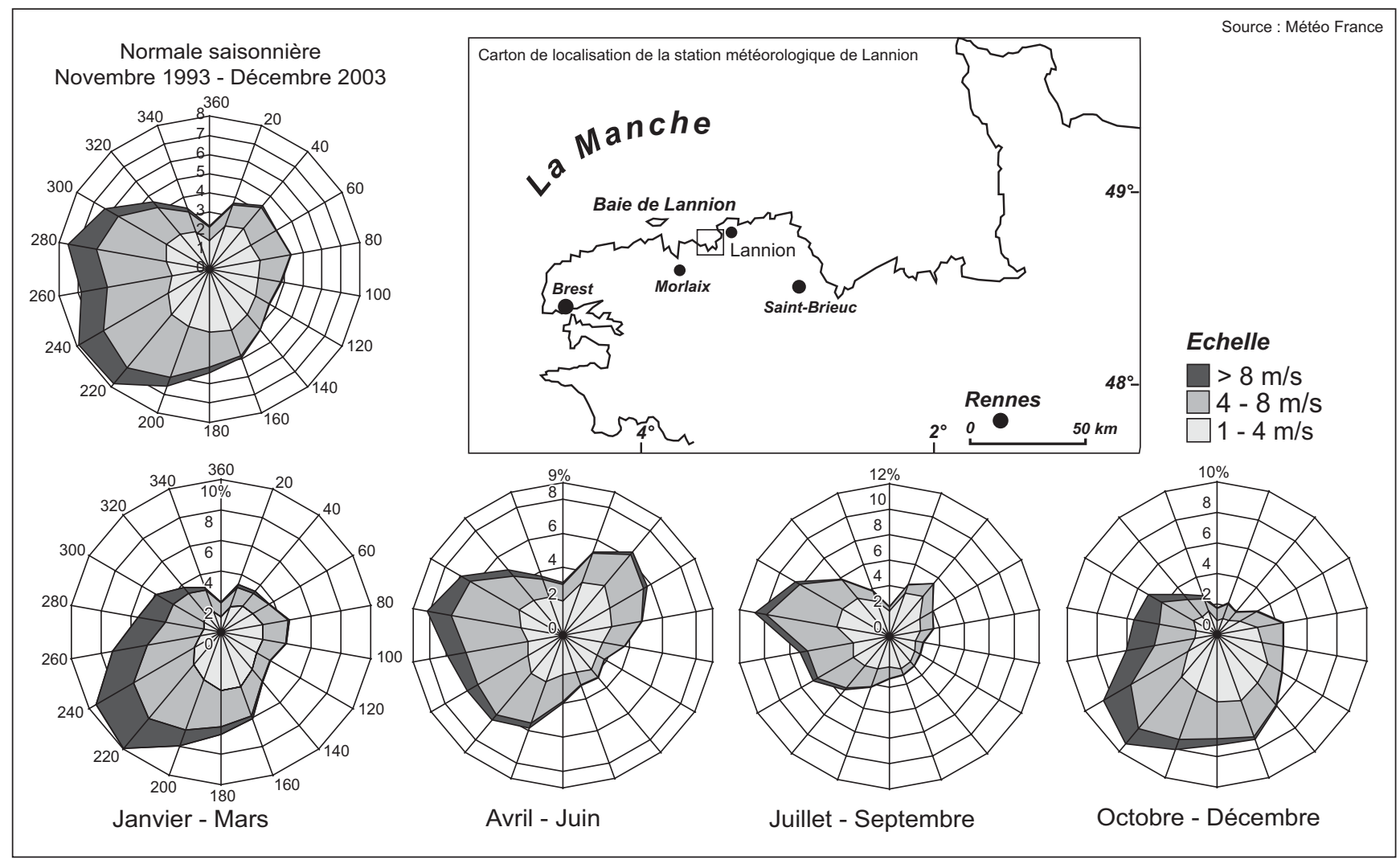

Fig. 4 - Conditions de vents sur la zone d'étude à partir des données enregistrées pour la station météorologique de Lannion. Fig. 4 - Wind conditions for the study area obtained from data recorded at the Lannion wind station.

\begin{tabular}{|l|c|c|c|c|}
\hline \multirow{2}{*}{} & \multicolumn{5}{|c|}{ Campagnes de mesures } \\
\cline { 2 - 5 } & Front de dune & Profils de dune & Front de dune & Profils de dune \\
\hline $10 / 10 / 02$ & Pas de relevé effectué & $X$ & $X$ & $X^{*},{ }^{* *}$ \\
\hline $23 / 10 / 02$ & $X$ & $X$ & $X$ & $X$ \\
\hline $15 / 11 / 02$ & $X$ & $X$ & $X$ \\
\hline $05 / 12 / 02$ & $X$ & $X$ & $X$ \\
\hline $15 / 01 / 03$ & $X$ & $X$ & $X$ & $X$ \\
\hline $28 / 01 / 03$ & $X$ & $X$ & $X$ \\
\hline $18 / 02 / 03$ & $X$ & $X$ & $X$ & $X$ \\
\hline $06 / 03 / 03$ & $X$ & $X$ & $X$ & $X$ \\
\hline $09 / 04 / 03$ & $X$ & $X$ & $X$ & $X$ \\
\hline $16-20 / 05 / 03$ & $X$ & $X$ & $X$ & $X$ \\
\hline $13 / 06 / 03$ & $X$ & $X$ & $X$ & $X$ \\
\hline $15 / 09 / 03$ & $X$ & $X$ & $X$ & $X$ \\
\hline
\end{tabular}

Tableau 1 - Inventaire des levés topo-morphologiques effectués entre les mois d'octobre 2002 et septembre 2003 (* à l'exception du profil 03 ; ** à l'exception du profil 06).

Table 1 - List of topographic surveys realised between October 2002 and September 2003 ( ${ }^{*}$ except profile 03; ${ }^{* *}$ except profile 06). 
Fig. 5 - Évolution du cordon dunaire du Grand Rocher entre le mois d'octobre 2002 et le mois de septembre 2003. Evolution du front de dune du Grand Rocher entre (A) les mois d'octobre 2002 et de février 2003 et entre (B) les mois de février 2003 et de septembre 2003.

Fig. 5 - Dunes of Grand Rocher evolution between October 2002 and September 2003. Dune of Grand Rocher evolution between $(A)$ October 2002 and February 2003; between (B) February and September 2003.

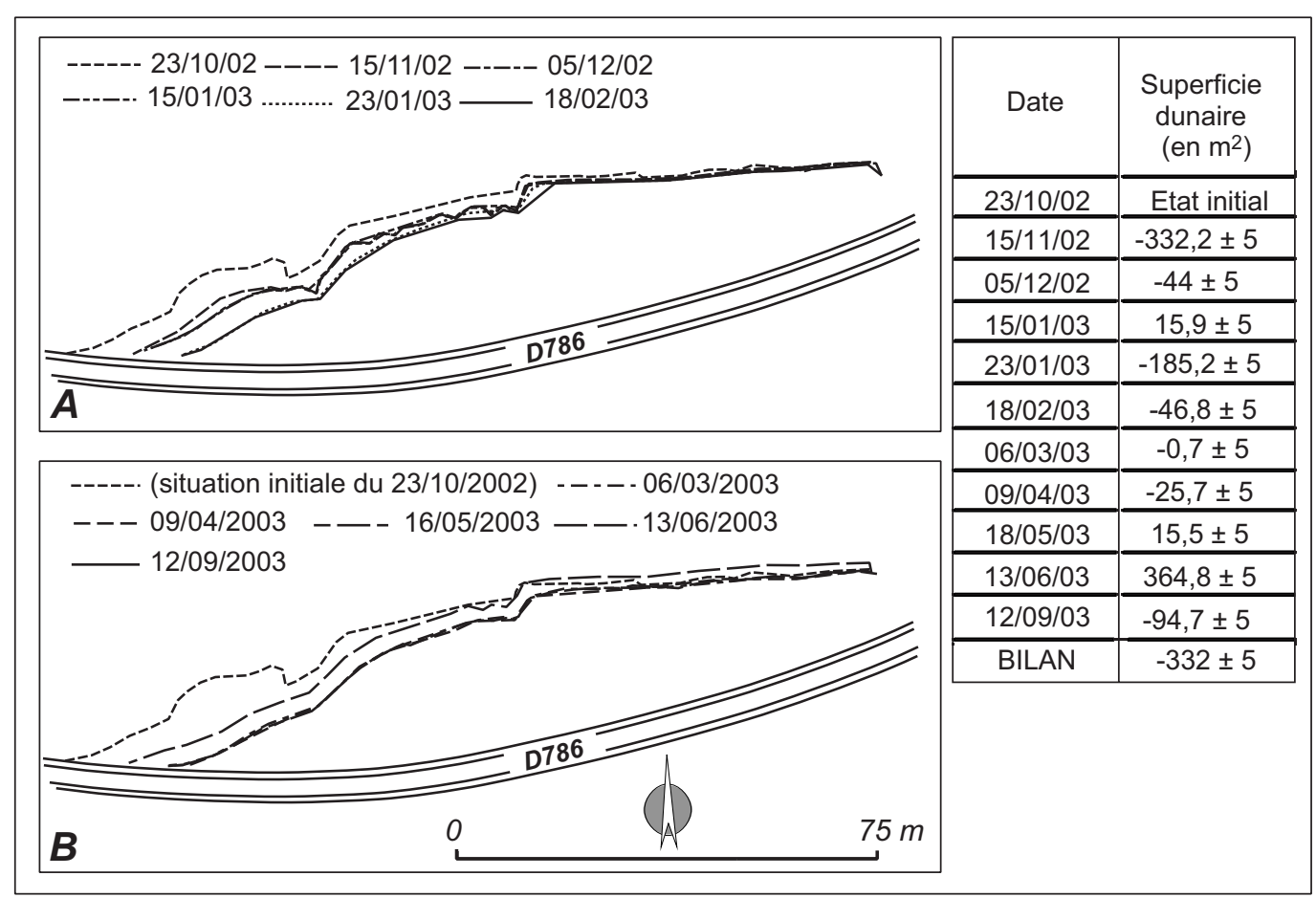

valeurs en $\mathrm{m}^{3} / \mathrm{m} .1$. Des marges d'erreur inhérentes à la mesure tachéométrique, calculées par méthode statistique, sont comprises entre 5 et $7 \mathrm{~cm}$ en $\mathrm{X}$ et $\mathrm{Y}$ et restent inférieures à $3 \mathrm{~cm}$ en $\mathrm{Z}$ (Suanez et al., 2005).

\section{Les résultats}

Nous séparerons les résultats acquis sur l'évolution du front de dune de ceux portant sur les profils de dune et du haut de plage.

\section{Mobilité du front de dune}

Pour le cordon dunaire du Grand Rocher, le bilan effectué au terme de la période d'observation montre un recul important du front de dune, la surface perdue atteignant $332 \pm$ $5 \mathrm{~m}^{2}$ (fig. 5). Cette évolution s'inscrit dans une tendance au recul qui est observée depuis 1999 (Suanez, 2004). Toutefois, c'est principalement entre les mois d'octobre et de mars 2003 que le recul s'effectue. Durant cette période, le cordon dunaire perd près de $618 \pm 5 \mathrm{~m}^{2}$, soit un quart de sa superficie initiale du mois d'octobre 2002 (2 $\left.725 \pm 5 \mathrm{~m}^{2}\right)$. Si l'érosion affecte l'ensemble de l'édifice, c'est toutefois la partie ouest qui reste la plus touchée. À certains endroits, la dune a totalement disparu découvrant ainsi l'enrochement qui est désormais battu par la houle. Dans le détail, les mesures montrent que le recul s'est effectué par à-coups, les mois de novembre 2002 et janvier 2003 enregistrant les pertes les plus importantes (respectivement $-330 \mathrm{~m}^{2}$ et $-185 \mathrm{~m}^{2}$ ). À partir du mois de mars 2003, la position du front de dune se stabilise dans un premier temps avant de connaître une phase de progradation importante durant l'été 2003. La surface gagnée durant cette période atteint plus de $365 \pm 5 \mathrm{~m}^{2}$.

À l'inverse du cordon dunaire du Grand Rocher, une grande stabilité du front de dune de Saint-Michel-en-Grève est observée (fig. 6). Entre les mois d'octobre 2002 et de mai 2003, les modifications restent mineures. L'évolution des superficies dunaires illustre ces changements peu importants. Au mois de mai 2003, la surface du cordon dunaire équivaut à celle du mois d'octobre 2002. Le levé effectué au mois de juin 2003 montre une augmentation très nette d'environ $1450 \pm 28 \mathrm{~m}^{2}$. Cette tendance se poursuit durant la période estivale car les mesures effectuées en septembre 2003 indiquent une nouvelle augmentation de la surface d'environ $1776 \pm 28 \mathrm{~m}^{2}$. Sur l'ensemble de la période, le cordon dunaire de Saint-Michel-en-Grève a gagné $3363 \pm 28 \mathrm{~m}^{2}$.

\section{Évolution du système " cordon dunaire / haut de plage »}

L'évolution du système « cordon dunaire / haut de plage » du Grand Rocher montre une évolution contrastée entre les profils 1 et 2 (fig. 7). Les bilans obtenus sur l'ensemble de la période montrent un déficit très important au niveau du profil 1 situé à l'ouest (- $\left.4 \mathrm{~m}^{3} / \mathrm{m} .1\right)$, alors qu'au niveau du profil 2 situé à l'est, le système s'est engraissé $\left(1,5 \mathrm{~m}^{3} / \mathrm{m} .1\right)$.

Dans le détail, on s'aperçoit que l'érosion a prédominé durant la période hivernale entre les mois d'octobre 2002 et de mars 2003. Une phase particulièrement érosive enregistrée au mois de janvier 2003 s'illustre par une déperdition de matériel sédimentaire atteignant respectivement $-2,5$ et $-1,5 \mathrm{~m}^{3} / \mathrm{m} .1$ pour les profils 1 et 2 (fig. 7). À partir de cette période, l'ensemble du système connaît un engraissement continu, particulièrement net dans la partie orientale du cordon dunaire (profil 2). Au niveau du profil 1, on note encore une phase érosive bien marquée durant le printemps 2003.

Le cordon dunaire de Saint-Michel-en-Grève connaît un engraissement sédimentaire continu tout au long de la période d'observation (fig. 8). Si quelques phases de démaigrissement apparaissent, à l'exemple du profil 3 à la date du 
Fig. 6 - Évolution du cordon dunaire de Saint-Michel-en-Grève entre le mois d'octobre 2002 et le mois de septembre 2003.

Fig. 6 - Evolution of the coastal dunes of Saint-Michel-en-Grève between October 2002 and September 2003.

18 février 2003, la tendance observée au niveau des quatre profils montre que le cordon s'engraisse de façon importante. Le bilan sédimentaire global calculé à partir des quatre radiales reste largement positif. Il est compris entre +1 et $+2,5 \mathrm{~m}^{3} / \mathrm{m} .1$ suivant les secteurs.

\section{Analyse des forçages météorologiques}

\section{Méthodes et données utilisées}

Dans le but de cerner l'impact des forçages météorologiques sur l'évolution morphosédimentaire des cordons dunaires, une analyse des données météo-marines sur la période d'observation a été effectuée afin de distinguer, d'une part, les événements tempétueux intervenant dans la dégradation des dunes et, d'autre part, les périodes d'accalmie généralement propices à la régénération des systèmes dunaires. Quatre types de données ont été retenus : les données de vent tri-horaires (vitesse et direction) pour la station de Lannion, la pression barométrique (valeurs tri-horaires pour la station de Lannion), la marée enregistrée à Roscoff et des données de houle obtenues à partir de simulation numérique effectuée avec le logiciel TOMAWAC (fig. 9).

\section{Les données de houles et états de mer}

Les données de houle pour l'ensemble de la période d'étude du 1er septembre 2002 au 30 septembre 2004 ont été acquises par simulation numérique à partir du modèle d'états de mer océanique du logiciel TOMAWAC dans le cadre du projet de construction d'un atlas numérique de houle le long des côtes Atlantique-Manche-Mer du Nord (collaboration entre Météo France, le Centre d'Etudes Techniques Maritimes et d'Études Fluviales (CETMEF), et LNHE-EDF). Les données de vent utilisées pour la modélisation proviennent de mesures satellitaires effectuées dans le cadre du projet ISLSCP (International Satellite Land Surface Climatology Project) NOAA-NCEP/Reanalysis2 (champs de vent à $10 \mathrm{~m}$ sur un pas de temps de $6 \mathrm{~h}$ et un pas d'espace de $1,875^{\circ}$ ). Les caractéristiques de houle ont été calculées en un point situé à l'entrée NW de la baie de Saint-Michel-en-Grève (coordonnées $3,7368^{\circ}$ de longitude $\mathrm{W}$ et $48,9294^{\circ}$ de latitude $\mathrm{N}$ ). Trois paramètres réduits du spectre directionnel de houle ont été calculés toutes les $3 \mathrm{~h}$ : la hauteur significative spectrale $\mathrm{H}_{\text {mo }}$ en mètre, la direction moyenne de propagation im en degré, la période de pic ( $\mathrm{T}_{\text {pic }}$ ) par la méthode de Read à l'ordre 5 en seconde.

Les données obtenues sur l'ensemble de la période d'étude montrent des hauteurs maximales d'environ $9 \mathrm{~m}$ associées

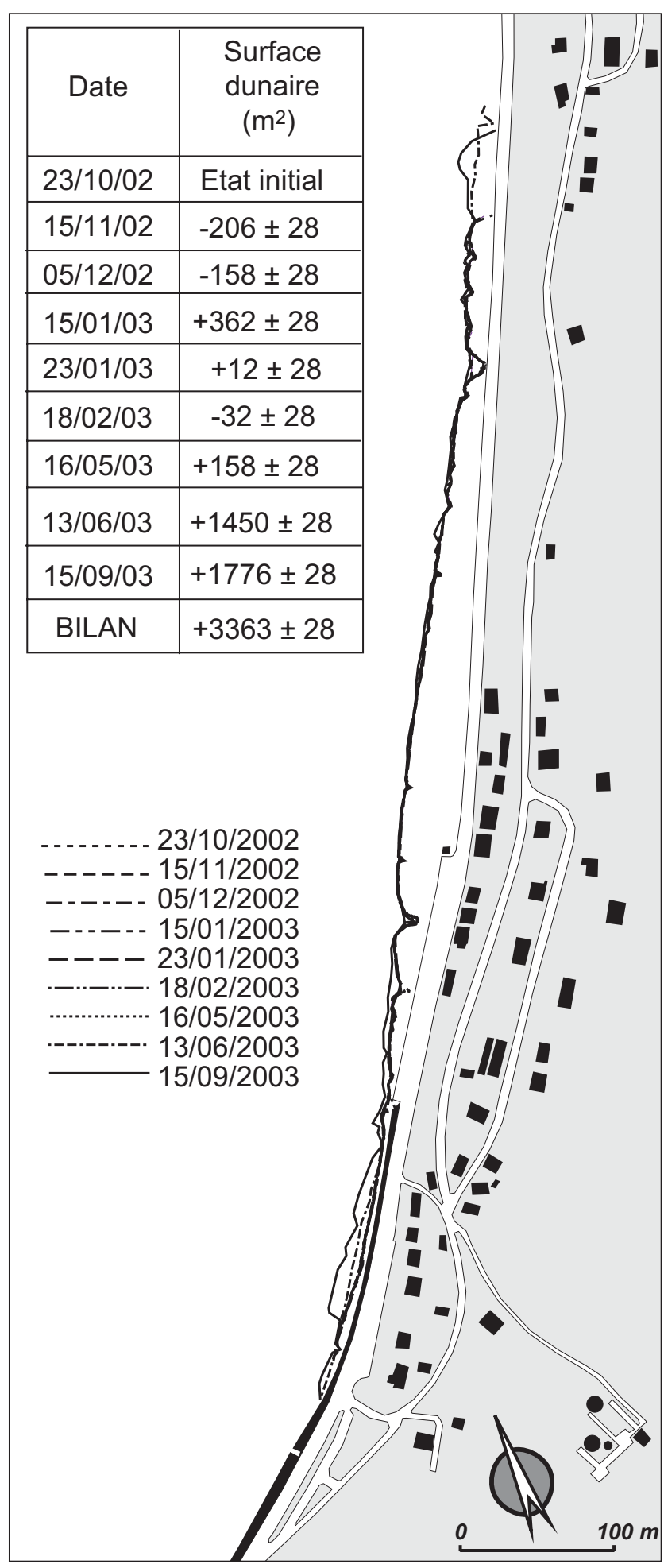

à des périodes de plus de 21 secondes. La hauteur moyenne est égale à $1,75 \mathrm{~m}$ pour une période de 10 secondes (tab. 2). La prise en compte des valeurs modales de H1/3 = $2 \mathrm{~m}$ et de $\mathrm{T} 1 / 3=9 \mathrm{~s}$, calculées à partir des mesures effectuées au large de Ouessant entre 1985 et 2001, et disponibles en libre accès sous la base de données Candhis du CETMEF (http:// www.cetmef.equipement.gouv.fr/donnees/candhis/), permet de cerner la période de forte agitation qui s'étend du mois d'octobre 2002 au mois d'avril 2003 (fig. 9). 


\section{Les niveaux d'eau extrêmes}

À l'exception des oscillations du plan d'eau liées à la marée astronomique, trois éléments principaux interviennent dans l'élévation « temporaire » du niveau marin à la côte définissant ainsi la notion de niveaux extrêmes et jouant dans l'érosion des littoraux, que ce soient les côtes à falaise ou les littoraux meubles (Shih et Komar, 1994 ; Ruggeiro et al., 1997, 2001).

Les surcotes, le plus souvent associées aux passages des perturbations atlantiques, s'expliquent par deux phénomènes (Bouligand et Pirazzoli, 1999 ; Pirazzoli, 2000 ; Betts et al., 2004 ; Pirazzoli et al., 2004) : une diminution de la pression barométrique (par définition < $1015 \mathrm{hpa}$ ) et l'intervention de vents de mer violents poussant la masse océanique à la côte.
L'élévation du niveau moyen de la mer liée à l'agitation, encore appelée le setup ou $\bar{\eta}_{\max }$, (Komar, 1998). Cette déformation du plan d'eau forme un angle dont la pente orientée vers le rivage trouve son origine au niveau de la zone de déferlement.

Enfin, les processus qui commandent la zone de swash interviennent également dans l'élévation du niveau marin au rivage. Cette dernière appelée run-up est définie comme étant la somme de deux composantes : les variations du plan d'eau liées à l'agitation (setup $=\bar{\eta}_{\max }$ ) et l'action du jet de rive (wave uprush $=\mathrm{R})$, soit $\mathrm{R}^{\mathrm{T}}=\bar{\eta}_{\max }+\mathrm{R}($ Komar, 1998 ; Ruggiero et al., 2001 ; Masselink et Hughes, 2003).

Dans le cas présent, l'analyse des niveaux extrêmes repose sur la quantification de deux paramètres, les surcotes et le run-up. Les surcotes ont été calculées par simple différence entre la marée observée et la marée prédite enregistrée par le

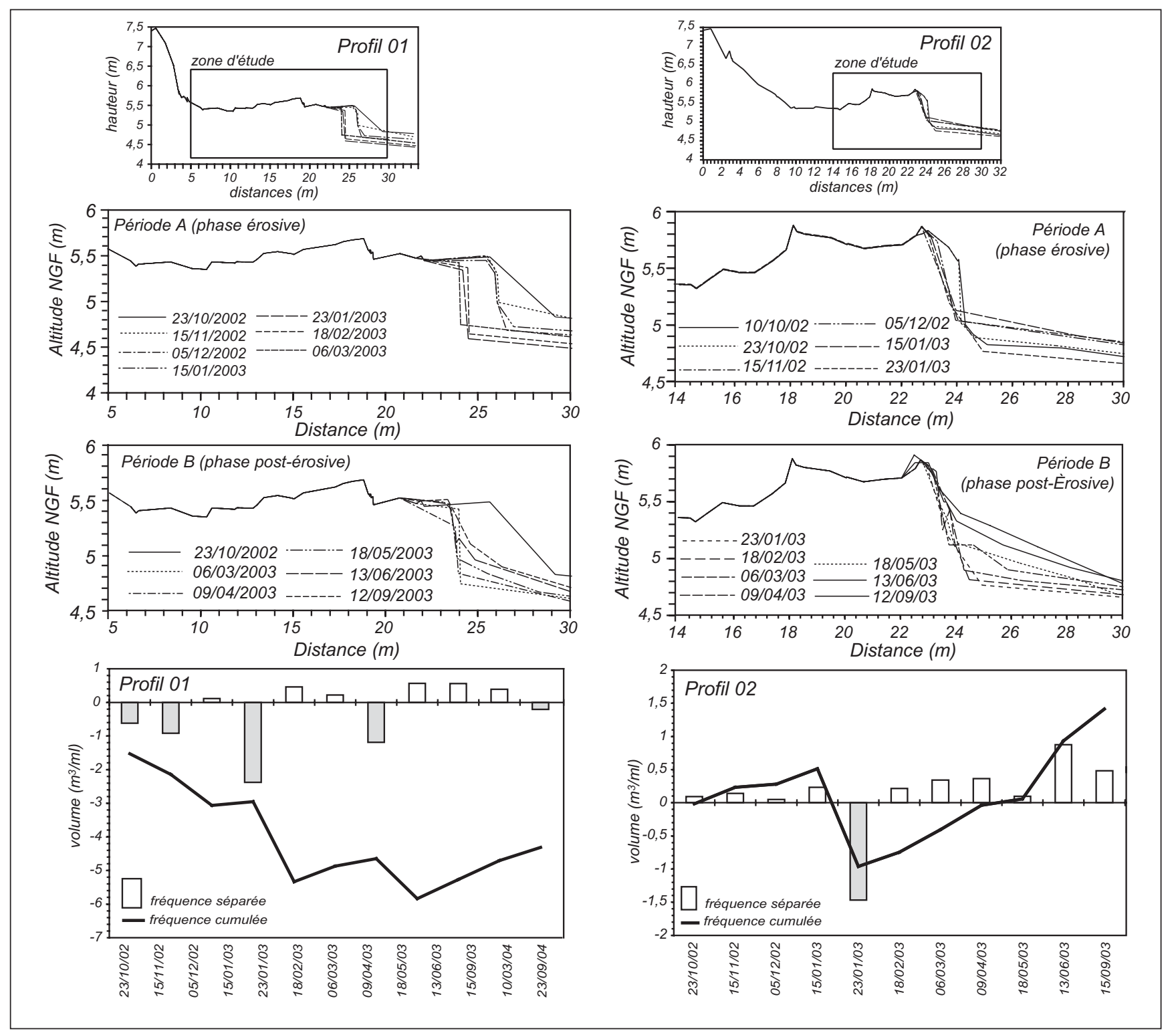

Fig. 7 - Évolution du bilan sédimentaire $\left(\mathrm{m}^{3} / \mathrm{m} . \mathrm{l}\right)$ du cordon dunaire du Grand Rocher mesurée à partir des profils 1 et 2.

Fig. 7 - Evolution of the sediment budget ( $\left.\mathrm{m}^{3} / \mathrm{l} . \mathrm{m}\right)$ of the dunes of Grand Rocher based on the surveys of profiles 1 and 2. 

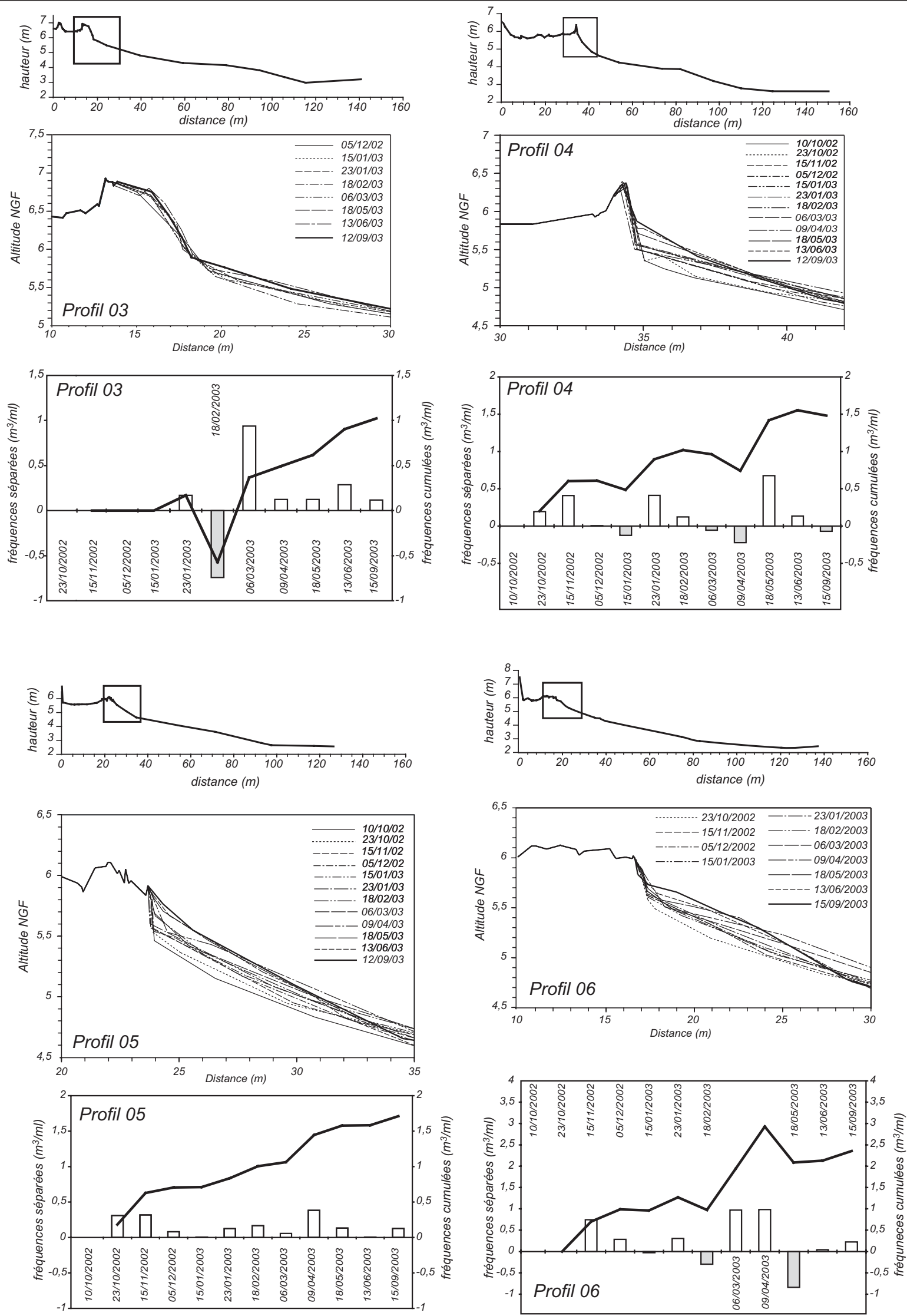

Fig. 8 - Évolution du bilan sédimentaire $\left(\mathrm{m}^{3} / \mathrm{m} . \mathrm{l}\right)$ du cordon dunaire de Saint-Michel-en-Grève mesurée à partir des profils $3,4,5$ et 6 . Fig. 8 - Evolution of the sediment budget $\left(\mathrm{m}^{3} / \mathrm{l} . \mathrm{m}\right)$ of the dunes of Saint-Michel-en-Grève based on the surveys of profiles $3,4,5$ and 6. 


\begin{tabular}{|c|c|c|c|c|c|c|}
\hline Profil & \multicolumn{2}{|r|}{$S(\tan \beta)$} & $\mathrm{H}_{\mathrm{mo}}(\mathrm{m})$ & $\mathrm{T}_{\text {pic }}(\mathrm{sec})$. & $\xi_{0}$ & $\mathbf{R}^{\mathbf{T}_{2 \%}}(\mathrm{~m})$ \\
\hline $\mathrm{N}^{\circ} 1$ & 0,012 & \multirow{2}{*}{$\begin{array}{c}\text { Grand Rocher : } \\
0,012\end{array}$} & \multirow{6}{*}{$\begin{array}{l}\min .0,23 \\
\text { moy. } 1,75\end{array}$} & \multirow{6}{*}{$\begin{array}{l}\min .2,9 \\
\operatorname{moy} .9,72 \\
\max .21,6\end{array}$} & $\min .0,05$ & $\min .0,07$ \\
\hline $\mathrm{N} \times 2$ & 0,012 & & & & $\max .0,35$ & $\max .1,92$ \\
\hline $\mathrm{N} \times 3$ & 0,025 & \multirow{4}{*}{$\begin{array}{l}\text { St-Michel-en- } \\
\text { Grève : 0;025 }\end{array}$} & & & \multirow{4}{*}{$\begin{array}{c}\min .0,1 \\
\operatorname{moy} .0,25 \\
\max .0,7\end{array}$} & \multirow{4}{*}{$\begin{array}{l}\min .0,10 \\
\operatorname{moy} .0,69 \\
\max .2,77\end{array}$} \\
\hline$N^{\circ} 4$ & 0,029 & & & & & \\
\hline$N^{\circ} 5$ & 0,03 & & & & & \\
\hline$N^{\circ} 6$ & 0,024 & & & & & \\
\hline
\end{tabular}

Tableau 2 - Caractéristiques morphodynamiques des secteurs du Grand Rocher et de Saint-Michel-en-Grève et calcul du run-up à partir de l'équation de P. Ruggiero et al. (2001).

Table 2 - Morphodynamic characteristics of the Grand Rocher and SaintMichel-en-Grève zones and runup calculation using the $P$. Ruggiero et al.'s formula (2001). marégraphe de Roscoff pour l'ensemble de la période considérée (fig. 9). Ces données ont été acquises auprès du SHOM (Brest) dans le cadre d'une convention de partenariat. Le run-up a été calculé à partir des équations issues des travaux de R.A. Holman (1986) et P. Nielsen et D.J. Hanslow (1991). Ces derniers insistent sur la relation entre l'élévation verticale maximale du run-up et les deux paramètres que sont la hauteur de la houle au large $\mathrm{H}_{\text {sig }}$ ou $\mathrm{H}_{\text {mo }}$ et le nombre d'Iribarren (surf similarity parameter):

$$
\frac{R^{T}}{H_{o}}=C \xi_{o}
$$

où $\mathrm{R}^{\mathrm{T}}$ : valeur du run-up étant la somme du setup et du runup $\left(\mathrm{R}^{\mathrm{T}}=\eta+\mathrm{R}\right) ; \mathrm{H}_{\mathrm{o}}=\mathrm{H}_{\mathrm{mo}}$ : hauteur significative spectrale $\mathrm{H}_{\mathrm{mo}}$ (en mètres) ; $\mathrm{C}$ : constante sans dimension ; $\xi_{\mathrm{o}}=$ nombre d'Iribarren (Battjes, 1974) =

$$
\xi_{o}=\frac{\tan \beta}{\left(H_{o} / L_{o}\right)^{1 / 2}}
$$

De façon générale la constante $\mathrm{C}$ qui revient le plus souvent dans la littérature est égale à 0,92 (Komar, 1998 ; Masselink et Hughes, 2003). Cela nous donne l'expression mathématique suivante :

$$
R^{T}{ }_{2 \%}=0,92 H_{o} \xi_{o}
$$

où $\mathrm{R}_{2}{ }_{2}$ correspond au run-up dépassé par $2 \%$ des plus hauts jets de rive.

Plus récemment, les travaux de P. Ruggiero et al. (2001) portant sur l'analyse du run-up dans un contexte de plage de type dissipant (Agate beach, Oregon) ont montré que la relation entre la pente $\mathrm{S}$, la longueur d'onde Lo et le run-up était bien plus significative. L'expression mathématique que ces auteurs proposent est la suivante :

$$
R^{T}{ }_{2 \%}=0,27 \sqrt{S H_{o} L_{o}}
$$

où $\mathrm{S}$ : pente de la plage $(\tan \beta) ; \mathrm{H}_{\mathrm{o}}$ : hauteur de la houle au large $\left(\mathrm{H}_{\mathrm{mo}}\right) ; \mathrm{L}_{\mathrm{o}}$ : longueur d'onde $=\mathrm{gT}^{2} / 2 \pi=1,561 \mathrm{~T}^{2}(\mathrm{~g}$ accélération de la pesanteur : $9,81 \mathrm{~m} / \mathrm{s}^{2} ; \mathrm{T}=\mathrm{T}_{\text {pic }}$ : période de la houle en seconde).

Dans le cadre de cette étude, l'équation 4 a été utilisée en tenant compte du caractère «hyper-dissipant » de notre zone d'étude. Les résultats obtenus sont présentés dans le tableau 2. Pour des pentes $\tan \beta$ respectives de 0,012 et de 0,025 pour les secteurs de Grand Rocher et de Saint-Michelen-Grève, les valeurs tri-horaires calculées sur l'ensemble de la période donnent un $\mathrm{R}^{\mathrm{T}}{ }_{2 \%}$ moyen égal à $0,48 \mathrm{~m}$ pour le secteur du Grand Rocher et de 0,69 m pour celui de SaintMichel-en-Grève, les valeurs maxima atteignant respectivement $1,92 \mathrm{~m}$ et $2,77 \mathrm{~m}$.

\section{Les données de vents et de pressions barométriques}

Les données de vents (vitesse et direction) et de pressions barométriques proviennent des enregistrements tri-horaires Météo France de la station de Lannion. L'une des difficultés est de déterminer une vitesse de vent critique à partir de laquelle se produit un événement morphogène tempétueux. Les nombreuses études réalisées sur les tempêtes, en domaine méditerranéen ou atlantique, suggèrent des vitesses de vent très variées (Lemasson et Régnauld, 1997 ; Bruzzi, 1996, 1998 ; Audran, 1999 ; Clabaut et al., 2000 ; MeurFerrec et Ruz, 2002 ; Hénaff, 2004). Les seuils fixés par les auteurs dépendent de l'échelle spatio-temporelle à laquelle ils travaillent. Les travaux réalisés sur de grands domaines géographiques à des échelles de temps pluri-décennales ne retiennent que les événements très exceptionnels où les vitesses de vent sont supérieures à $15 \mathrm{~m} / \mathrm{s}$. À l'inverse, les études menées sur l'impact des événements épisodiques sur des secteurs plus restreints utilisent un seuil beaucoup plus bas, compris généralement entre 8 et $11 \mathrm{~m} / \mathrm{s}$. Dans le cadre de cette étude, nous considérons que, au-delà de $10 \mathrm{~m} / \mathrm{s}$, un événement potentiellement morphogène peut prendre place.

\section{Analyse des données}

La figure 9 synthétise l'ensemble des données météo-marines enregistrées sur l'ensemble de la période d'étude. Entre le 7 octobre 2002 et le 15 avril 2003, on note une forte activité morphogène. Treize épisodes définis comme étant potentiellement morphogènes ont été recensés (tab. 3). Ils présentent des caractéristiques hydrodynamiques et météomarines très variables. Certaines tempêtes, comme celles du 13 octobre 2002, 29 décembre 2002, 28 février 2003, 19 avril 2003 et du 2 mai 2003 coïncident avec de fortes marées (niveaux marégraphiques observés $>3,5 \mathrm{~m}$ ) et/ou de fortes surcotes pouvant dépasser $30 \mathrm{~cm}$. À l'inverse, les hauteurs de houle $\mathrm{H}_{\text {mo }}$ enregistrées durant ces événements ont été peu élevées (entre 3 et $4 \mathrm{~m}$ ). Dans d'autres cas, des caractéristiques inverses sont observées. Les épisodes morphogènes du 27 octobre 2002, du 2 décembre 2002 et du 
30 janvier 2003 sont marqués par des hauteurs de houles très importantes $\left(\mathrm{H}_{\mathrm{mo}}\right.$ compris entre 6 et $9 \mathrm{~m}$ ) ; à l'inverse les niveaux d'eau enregistrés durant ces événements sont peu élevés (faible marée observée et/ou faible surcote). Notons enfin que, à l'exception des épisodes morphogènes (toutefois de faible intensité) du 19 avril et du 2 mai 2003, une longue période d'accalmie prend place dès le mois de mars 2003. Ces conditions climatiques « exceptionnelles » marquées par un régime anticyclonique de beau temps se sont accompagnées le plus souvent de vents de secteurs est à nord-est.

\section{Analyse de forçages et des dynamiques morphosédimen- taires}

\section{Aspect méthodologique}

L'analyse des forçages météomarins et des dynamiques morphosédimentaires repose sur un traitement statistique permettant de corréler l'évolution diachronique des bilans sédimentaires aux conditions de forçage météo-marines enregistrées entre chaque campagne de mesure. À cet effet, l'Analyse factorielle en Composante Principale (ACP) a été utilisée. L'ACP mesure et représente séparément les relations entre les individus et celles entre les variables, sous la forme de deux nuages de points, dans un espace à n-1 dimensions défini par

Fig. 9 - Caractéristiques des conditions météo-marines enregistrées durant la période d'observation (octobre 2002 - septembre 2003).

Fig. 9 - Meteorological and marine conditions recorded during the period of survey (October 2002 - September 2003).

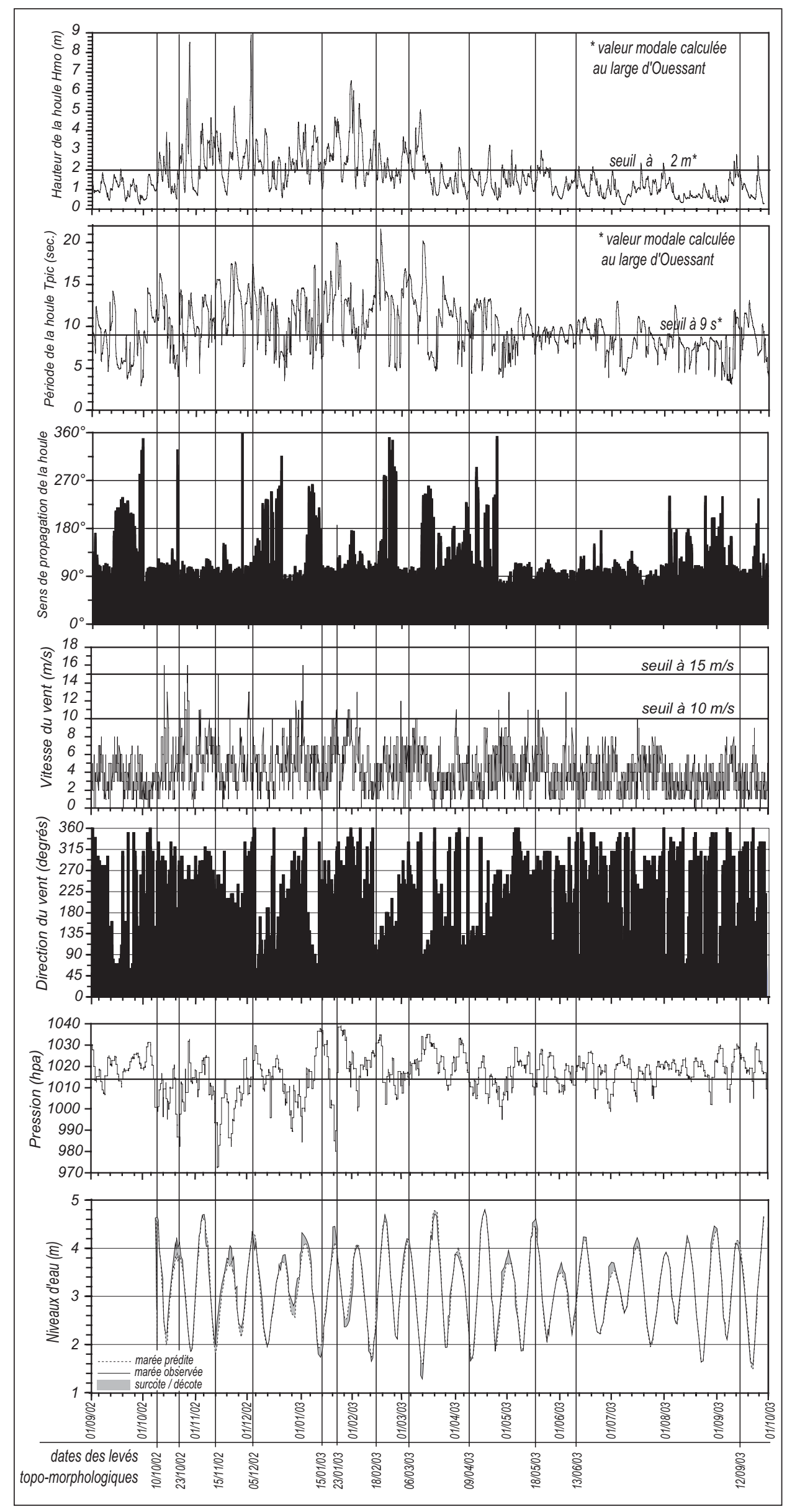




\begin{tabular}{|c|c|c|c|c|c|}
\hline $\begin{array}{l}\text { Dates et heures de } \\
\text { l'épisode }\end{array}$ & Hauteur houle $\mathrm{H}_{\mathrm{mo}}$ & Direction houle & Vitesse vent Vt & $\begin{array}{c}\text { Pression } \\
\text { barométrique }\end{array}$ & \begin{tabular}{|c} 
Surcotes / décotes et \\
niveau de pleine mer \\
observée
\end{tabular} \\
\hline \multicolumn{6}{|c|}{ Levé topo-morphologique du 10/10/2002 (état initial) } \\
\hline 14/10/2002 (0 h) & $3,92 \mathrm{~m}$ & $101^{\circ}$ & $9 \mathrm{~m} / \mathrm{s}$ & 1009 hpa & $5 \mathrm{~cm}-\mathrm{PM}: 2,73 \mathrm{~m}$ \\
\hline \multicolumn{6}{|c|}{ Levé topo-morphologique du 23/10/2002 } \\
\hline $27 / 10 / 2002(10 \mathrm{~h})$ & $8,5 \mathrm{~m}$ & $100^{\circ}$ & $16 \mathrm{~m} / \mathrm{s}$ & $1008 \mathrm{hpa}$ & $3 \mathrm{~cm}-\mathrm{PM}: 2,88 \mathrm{~m}$ \\
\hline 08/11/2002 (09 h) & $4,67 \mathrm{~m}$ & $95^{\circ}$ & $10 \mathrm{~m} / \mathrm{s}$ & $1006 \mathrm{hpa}$ & $-7 \mathrm{~cm}-\mathrm{PM}: 4,03 \mathrm{~m}$ \\
\hline 12/11/2002 (13 h) & $3,7 \mathrm{~m}$ & $98^{\circ}$ & $15 \mathrm{~m} / \mathrm{s}$ & 972 hpa & $16 \mathrm{~cm}-\mathrm{PM}: 2,26 \mathrm{~m}$ \\
\hline \multicolumn{6}{|c|}{ Levé topo-morphologique du 15/11/2002 } \\
\hline $02 / 12 / 2002(05 \mathrm{~h})$ & $8,9 \mathrm{~m}$ & $110^{\circ}$ & $13 \mathrm{~m} / \mathrm{s}$ & $1007 \mathrm{hpa}$ & $8 \mathrm{~cm}-\mathrm{PM}: 3,75 \mathrm{~m}$ \\
\hline \multicolumn{6}{|c|}{ Levé topo-morphologique du 05/12/2002 } \\
\hline 29/12/2002 (15 h) & $4,45 \mathrm{~m}$ & $85^{\circ}$ & $12 \mathrm{~m} / \mathrm{s}$ & $1003 \mathrm{hpa}$ & $27 \mathrm{~cm}-\mathrm{PM}: 3,10 \mathrm{~m}$ \\
\hline \multicolumn{6}{|c|}{ Levé topo-morphologique du 15/01/2003 } \\
\hline 20/01/2003 (08 h) & $4,48 \mathrm{~m}$ & $76^{\circ}$ & $10 \mathrm{~m} / \mathrm{s}$ & 990 hpa & $42,5 \mathrm{~cm}-\mathrm{PM}: 4,42 \mathrm{~m}$ \\
\hline \multicolumn{6}{|c|}{ Levé topo-morphologique du 23/01/2003 } \\
\hline $30 / 01 / 2003(18$ h) & $6,05 \mathrm{~m}$ & $168^{\circ}$ & $10 \mathrm{~m} / \mathrm{s}$ & $1015 \mathrm{hpa}$ & $-30 \mathrm{~cm}-\mathrm{PM}: 2,82 \mathrm{~m}$ \\
\hline 03/02/2002 (08 h) & $5,3 \mathrm{~m}$ & $109^{\circ}$ & $9 \mathrm{~m} / \mathrm{s}$ & 1005 hpa & 1,4 cm - PM : 4,07 m \\
\hline \multicolumn{6}{|c|}{ Levé topo-morphologique du 18/02/2003 } \\
\hline 28/02/2003 (18 h) & $3,7 \mathrm{~m}$ & $84^{\circ}$ & $10 \mathrm{~m} / \mathrm{s}$ & $1012 \mathrm{hpa}$ & $28 \mathrm{~cm}-\mathrm{PM}: 3,21 \mathrm{~m}$ \\
\hline \multicolumn{6}{|c|}{ Levé topo-morphologique du 06/03/2003 } \\
\hline 10/03/2003 (19 h) & $5,07 \mathrm{~m}$ & $102^{\circ}$ & $7 \mathrm{~m} / \mathrm{s}$ & 1022 hpa & $-3 \mathrm{~cm}-\mathrm{PM}: 2,46 \mathrm{~m}$ \\
\hline \multicolumn{6}{|c|}{ Levé topo-morphologique du 09/04/2003 } \\
\hline 19/04/2003 (21 h) & $3,27 \mathrm{~m}$ & $222^{\circ}$ & $8 \mathrm{~m} / \mathrm{s}$ & 1009 hpa & $6 \mathrm{~cm}-\mathrm{PM}: 4,48 \mathrm{~m}$ \\
\hline 02/05/2003 (08 h) & $3,02 \mathrm{~m}$ & $84^{\circ}$ & $13 \mathrm{~m} / \mathrm{s}$ & 1004 hpa & $31 \mathrm{~cm}-\mathrm{PM}: 3,92 \mathrm{~m}$ \\
\hline \multicolumn{6}{|c|}{$\begin{array}{l}\text { Levé topo-morphologique du } 18 / 05 / 2003 \\
\text { Levé topo-morphologique du } 13 / 06 / 2003 \\
\text { Levé topo-morphologique du } 12 / 09 / 2003\end{array}$} \\
\hline
\end{tabular}

Tableau 3 - Caractéristiques hydrodynamiques et météo-marines des principaux épisodes morphogènes enregistrés durant la période d'observation.

Table 3 - Atmospheric and hydrodynamic characteristics of the main geomorphic events recorded during the period of survey.

n-1 axes factoriels (Chamussy et al., 1994). Les fortes relations s'expriment selon la distance entre les points qui, dans le cas de l'ACP, est une distance euclidienne. Les axes factoriels sont hiérarchisés de telle sorte qu'ils comportent une part décroissante d'information, encore appelée « inertie ». On considère qu'au-delà du deuxième axe factoriel, la part d'information retenue par le modèle n'est plus significative.

Dans le cas qui nous occupe, deux dynamiques morphosédimentaires sont analysées : les phases d'érosion et de régénération des cordons dunaires. Pour les phases d'érosion, nous recherchons les relations les plus fortes qui permettent d'identifier le rôle des différents agents météomarins dans la diminution du bilan sédimentaire des cordons dunaires : les superficies dunaires (SUPDU1 pour le cordon dunaire du Grand Rocher ; SUPDU2 pour celui de SaintMichel-en-Grève) et les surfaces verticales calculées sur les six profils (SUPROF1, SUPROF2, SUPROF3, etc.). Notre attention a été portée sur l'importance des niveaux extrêmes comme facteur explicatif de l'érosion des dunes. Ainsi, cinq variables ont été retenues, la hauteur de la houle (Hmo), la marée observée (MAROBS), qui fait la somme du niveau marin donné par la marée prédite et de la surcote, le run-up (RUNUP1 pour le cordon dunaire du Grand Rocher ; RUNUP2 pour celui de Saint-Michel-en-Grève), la vitesse du vent (VITV) et la pression barométrique (PRESS).

Les valeurs ayant été retenues pour chaque variable correspondent à celles qui caractérisent l'épisode le plus morphogène enregistré entre chaque campagne de mesure. Cette discrimination comporte une part d'incertitude car, comme nous l'avons vu précédemment, deux à trois épisodes tempétueux ont pu avoir lieu entre deux relevés topo-morphologiques, comme cela s'est produit durant les périodes d'octobre à novembre, de janvier à février et d'avril à mai (tab. 3). Dans tous les cas, notre choix s'est fait en fonction de deux paramètres essentiels, les plus importantes hauteurs d'eau de marée observée et/ou les plus fortes 
Tableau 4 - Dates, heures et caractéristiques des épisodes morphogènes retenus pour l'analyse factorielle en composante principale.

Table 4 - Dates, hours, and characteristics of the geomorphic storm events selected for the PCA statistical analysis (Principal Component Analysis).

hauteurs de houle. Le tableau 4 consigne les épisodes morphogènes retenus. Pour la période allant du mois de mai à septembre 2003, marquée par une absence de tempête, nous avons sélectionné « l'épisode » caractérisé par une hauteur de houle et/ou un niveau marégraphique le(s) plus important(s).

Pour l'analyse des phases de régénération des cordons dunaires, nous avons privilégié une approche théorique en travaillant sur les périodes durant lesquelles les vents de mer ont pu générer un engraissement du système haut de plage / cordon dunaire. Une quantification des volumes de sables transportés par le vent a été réalisée pour chaque intervalle de temps séparant deux levés (tab. 5). Pour ce faire, une dis-

\begin{tabular}{|c|c|c|}
\hline Périodes & Episodes morphogènes & $\begin{array}{c}\mathbf{H}_{\text {mo }} \text { - hauteur } \\
\text { de la marée observée }\end{array}$ \\
\hline $10 / 10 / 02-23 / 10 / 02$ & $14 / 10 / 2002(0 \mathrm{~h})$ & $3,92 \mathrm{~m}-2,73 \mathrm{~m}$ \\
\hline $23 / 10 / 02-15 / 11 / 02$ & $27 / 10 / 2002(10 \mathrm{~h})$ & $8,5 \mathrm{~m}-2,88 \mathrm{~m}$ \\
\hline $15 / 11 / 02-05 / 12 / 02$ & $02 / 12 / 2002(05 \mathrm{~h})$ & $8,9 \mathrm{~m}-3,75 \mathrm{~m}$ \\
\hline $05 / 12 / 02-15 / 01 / 03$ & $29 / 12 / 2002(15 \mathrm{~h})$ & $4,45 \mathrm{~m}-3,10 \mathrm{~m}$ \\
\hline $15 / 01 / 03-23 / 01 / 03$ & $20 / 01 / 2003(08 \mathrm{~h})$ & $4,48 \mathrm{~m}-4,42 \mathrm{~m}$ \\
\hline $23 / 01 / 03-18 / 02 / 03$ & $03 / 02 / 2003(08 \mathrm{~h})$ & $5,3 \mathrm{~m}-4,07 \mathrm{~m}$ \\
\hline $18 / 02 / 03-06 / 03 / 03$ & $28 / 02 / 2003(18 \mathrm{~h})$ & $3,7 \mathrm{~m}-3,21 \mathrm{~m}$ \\
\hline $06 / 03 / 03-09 / 04 / 04$ & $10 / 03 / 2003(19 \mathrm{~h})$ & $5,07 \mathrm{~m}-2,46 \mathrm{~m}$ \\
\hline $09 / 04 / 03-18 / 05 / 03$ & $19 / 04 / 2003(09 \mathrm{~h})$ & $3,27 \mathrm{~m}-4,48 \mathrm{~m}$ \\
\hline $18 / 05 / 03-13 / 06 / 03$ & $10 / 06 / 2003(15 \mathrm{~h})$ & $1,98 \mathrm{~m}-2,98 \mathrm{~m}$ \\
\hline $13 / 06 / 03-12 / 09 / 03$ & $10 / 09 / 2003(19 \mathrm{~h})$ & $2,45 \mathrm{~m}-4,05 \mathrm{~m}$ \\
\hline
\end{tabular}

crimination des vents morphogènes a été effectuée à partir de deux filtres : la vitesse d'arrachement des sables et la direction qui est fonction de l'orientation et de la localisation des cordons dunaires : $280^{\circ}$ à $80^{\circ}$ nord pour le cordon dunaire du Grand Rocher et $220^{\circ}$ à $20^{\circ}$ nord pour celui de Saint-Michel-en-Grève. Les vitesses d'arrachement des sables ont été retenues dès lors que la vitesse du vent mesurée $\mathrm{V}^{*}>\mathrm{V}^{*}{ }_{\mathrm{c}}$ (vitesse critique d'arrachement des sables).

\begin{tabular}{|c|c|c|c|c|c|c|c|}
\hline \multirow{2}{*}{$\begin{array}{l}\text { Périodes correspondant } \\
\text { aux intervalles de temps } \\
\text { entre chaque levé }\end{array}$} & \multirow{2}{*}{$\begin{array}{l}\text { Temps après } \\
\text { filtrage sur } \\
\text { la vitesse et } \\
\text { la direction } \\
\text { (Grand Rocher) }\end{array}$} & \multicolumn{2}{|c|}{$\begin{array}{l}\text { Volume Grand } \\
\text { Rocher }\left(\mathrm{m}^{3} / \mathrm{m} . \mathrm{l}\right)\end{array}$} & \multirow{2}{*}{$\begin{array}{c}\text { Temps après } \\
\text { filtrage sur la } \\
\text { vitesse et la } \\
\text { direction (St- } \\
\text { Michel-en-Grève) }\end{array}$} & \multicolumn{2}{|c|}{$\begin{array}{l}\text { Volume St-Michel- } \\
\text { en-Grève }\left(\mathrm{m}^{3} / \mathrm{m} . \mathrm{l}\right)\end{array}$} & \multirow{2}{*}{\begin{tabular}{|c} 
Total des \\
précipitations (mm) \\
et précipitations / jour \\
$(\mathrm{mm})$
\end{tabular}} \\
\hline & & Eq. (6) & Eq. (7) & & Eq. (6) & Eq. (7) & \\
\hline $10 / 10 / 02-23 / 10 / 02$ & $51 \mathrm{~h}$ & 0,26 & 0,55 & $93 \mathrm{~h}$ & 0,61 & 1,38 & $21,6(1,5)$ \\
\hline $23 / 10 / 02-15 / 11 / 02$ & $63 \mathrm{~h}$ & 0,23 & 0,45 & $231 \mathrm{~h}$ & 1,42 & 3,14 & $36,2(1,6)$ \\
\hline $15 / 11 / 02-05 / 12 / 02$ & $63 \mathrm{~h}$ & 0,30 & 0,70 & $81 \mathrm{~h}$ & 0,48 & 1,07 & $21,6(1,1)$ \\
\hline 05/12/02 - 15/01/03 & $132 \mathrm{~h}$ & 0,30 & 0,50 & $162 \mathrm{~h}$ & 0,80 & 1,80 & $36,4(0,9)$ \\
\hline $15 / 01 / 03-23 / 01 / 03$ & $30 \mathrm{~h}$ & 0,05 & 0,06 & $66 \mathrm{~h}$ & 0,23 & 0,45 & $14(1,75)$ \\
\hline $23 / 01 / 03-18 / 02 / 03$ & $138 \mathrm{~h}$ & 0,61 & 1,28 & $204 \mathrm{~h}$ & 0,82 & 1,68 & $24,8(1)$ \\
\hline $18 / 02 / 03-06 / 03 / 03$ & $6 \mathrm{~h}$ & 0,01 & 0,01 & $24 \mathrm{~h}$ & 0,04 & 0,05 & $12,8(0,75)$ \\
\hline 06/03/03 - 09/04/04 & $117 \mathrm{~h}$ & 0,19 & 0,24 & $81 \mathrm{~h}$ & 0,24 & 0,45 & $7,2(0,2)$ \\
\hline 09/04/03 - 18/05/03 & $78 \mathrm{~h}$ & 0,20 & 0,36 & $126 \mathrm{~h}$ & 0,54 & 1,14 & $26(0,67)$ \\
\hline $18 / 05 / 03-13 / 06 / 03$ & $24 \mathrm{~h}$ & 0,05 & 0,08 & $111 \mathrm{~h}$ & 0,42 & 0,85 & $15,6(0,56)$ \\
\hline $13 / 06 / 03-12 / 09 / 03$ & $147 \mathrm{~h}$ & 0,21 & 0,24 & $276 \mathrm{~h}$ & 0,48 & 0,65 & $39,8(0,45)$ \\
\hline
\end{tabular}

Tableau 5 - Calcul théorique du transit éolien orienté vers les cordons dunaires durant la période d'observation à partir des formules de R.A. Bagnolds (Eq. 6) et de R. Kawamura (Eq. 7). Total des précipitations ( $\mathrm{mm}$ ) tombées dans l'intervalle de temps et précipitations $(\mathrm{mm})$ ramenées à la journée pour chaque période.

Table 5 - Theoretical calculation of eolian sand transport to the dunes during the period of survey using R.A. Bagnolds's (Eq.6) and R. Kawamura's formulas (Eq.7). Total rainfall recorded for each interval of time and rainfall per day for each period. 
Fig. 10 - Analyse statistique en composantes principales visant à corréler le bilan sédimentaire des cordons dunaires et les conditions météo-marines enregistrées pendant la période d'observation.

Fig. 10 - Statistical analysis (PCA) of dune sediment budget and meteo-marine conditions recorded during the monitoring period.
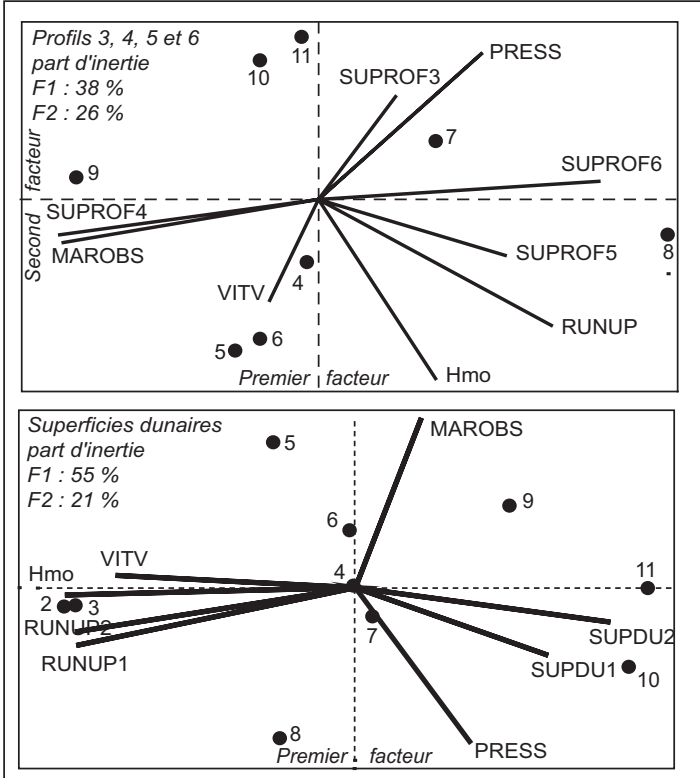

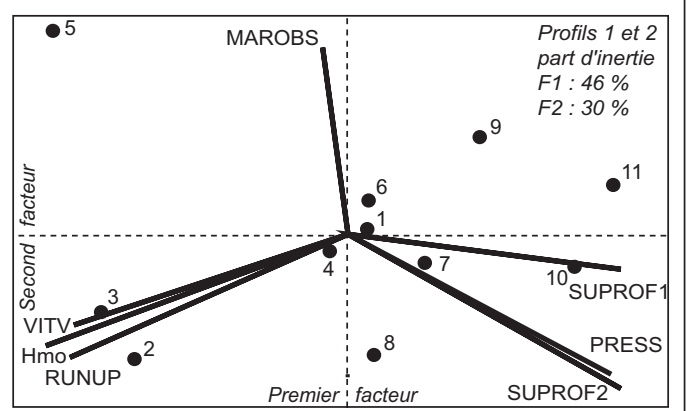

$1: 10$ octobre 2002 - 23 octobre 2002

2: 23 octobre 2002 - 15 novembre 2002

$3: 15$ novembre 2002 - 05 décembre 2002

4 : 05 décembre 2002 - 15 janvier 2003

$5: 15$ janvier 2003 - 23 janvier 2003

$6: 23$ janvier 2003 - 18 février 2003

$7: 18$ février 2003 - 06 mars 2003

8 : 06 mars 2003 - 09 avril 2003

9: 09 avril 2003 - 18 mai 2003

$10: 18$ mai $2003-13$ juin 2003

$11: 13$ juin 2003 - 12 septembre 2003
Dans le cas présent, la vitesse critique d'arrachement $\mathrm{V}_{\mathrm{c}}{ }_{\mathrm{c}}$ a été calculée à partir de la formule de R.A. Bagnold (1941) :

$$
V^{*}{ }_{\mathrm{c}}=k_{1}[(\rho s / \rho a)(g d)]^{0,5}
$$

où $\mathrm{k}_{1}$ est une constante $=0,1 ; \rho \mathrm{s}$ : densité des sables $\left(2,7 \mathrm{~g} / \mathrm{cm}^{3}\right) ; \rho a$ : densité de l'air $\left(0,0023 \mathrm{~g} / \mathrm{cm}^{3}\right) ; \mathrm{d}:$ diamètre $\mathrm{du}$ grain moyen en $\mathrm{cm} ; \mathrm{g}$ : accélération de la pesanteur $\left(9,81 \mathrm{~m} / \mathrm{s}^{2}\right)$. Pour un grain moyen de $155 \mu \mathrm{m}$, constituant les sables vifs du système haut de plage / dune, la vitesse critique d'arrachement $\mathrm{V}^{*}{ }_{\mathrm{c}}$ est égale à $5,7 \mathrm{~m} / \mathrm{s}$. Un seuil de $6 \mathrm{~m} / \mathrm{s}$ a été choisi pour filtrer les données brutes.

Les formules de quantification ayant été retenues sont celles de R.A. Bagnolds (1941) et de R. Kawamura (1964). Ces équations sont les plus fréquemment utilisées dans les études portant sur le transit sédimentaire éolien (Svasek et Terwindt, 1974 ; Sarre, 1988 ; Arens, 1994 ; Meur-Ferrec et Ruz, 2002 ; Alcantara-Carrio et Alonso, 2002) : équation de R.A. Bagnolds (1941)

$$
Q=C(d / D) 0,5 V_{c}^{*}(\rho a / g)
$$

équation de R. Kawamura, (1964)

$$
Q=K(\rho a / g)\left(V^{*}-V^{*}{ }_{c}\right)\left(V^{*}+V^{*}{ }_{c}\right)^{2}
$$

où $\mathrm{Q}$ : quantité de sable transporté $(\mathrm{kg} / \mathrm{m} / \mathrm{s}) ; \mathrm{V}^{*}$ : vitesse du vent mesuré en $\mathrm{cm} / \mathrm{s} ; \mathrm{V}_{\mathrm{c}}^{*}$ : vitesse critique d'arrachement en $\mathrm{cm} / \mathrm{s} ; \mathrm{C}$ : coefficient sans dimension qui dépend du tri du sédiment $=1,5$ très bon tri, 1,8 bon tri, 2,8 mauvais tri, 3,5 éléments non mobilisables ; $\mathrm{K}$ : constante de 2,7 pour des sables compris entre 100 et $800 \mu \mathrm{m}$; $\rho \mathrm{a}$ : densité de l'air $\left(1,23 \mathrm{~kg} / \mathrm{m}^{3}\right) ; \mathrm{g}$ : accélération de la pesanteur $\left(9,81 \mathrm{~m} / \mathrm{s}^{2}\right)$; $\mathrm{d}$ : le diamètre moyen des grains de sables (en $\mathrm{cm}$ ) et $\mathrm{D}$ : valeur du grain standard $(0,025 \mathrm{~cm})$.

\section{Résultats}

La figure 10 montre une bonne relation entre les fortes houles de tempête, les hauts niveaux de submersion qui leur sont associés (run-up) et l'érosion des cordons dunaires. Ces phénomènes sont visibles sur le terrain (fig. 11). Cette relation structure pleinement le premier facteur se rapportant à l'analyse des superficies dunaires (fig. 10A). Elle illustre

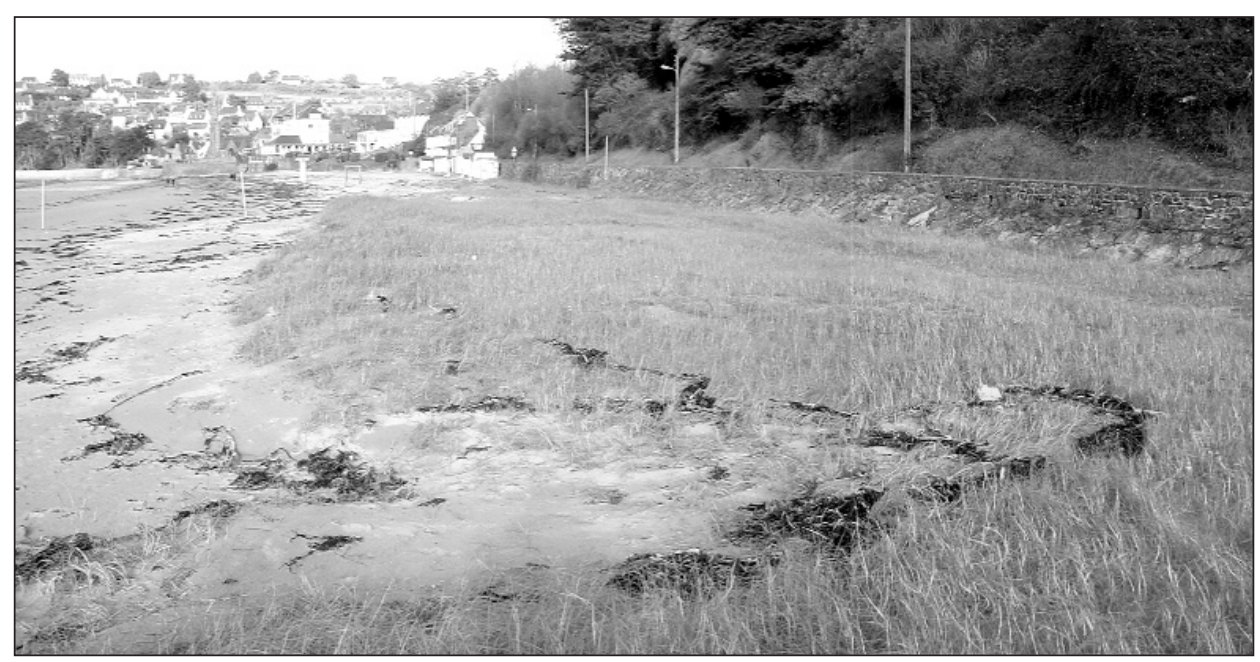
également la forte saisonnalité du fonctionnement morphosédimentaire en opposant la période allant de la fin du mois d'octobre au début du mois de décembre 2002, marquée, comme

Fig. 11 - Effets du run-up et submersion du cordon dunaire de Saint-Michel-en-Grève (cliché : 15/11/ 2002, S. Suanez).

Fig. 11 - Effects of run-up on the submergence of the coastal dune of Saint-Michel-en-Grève dune (photo: 15/11/2002, S. Suanez). 
on l'a vu, par des tempêtes fortement morphogènes à celle allant de la mi-mai au début du mois de septembre 2003, caractérisée en revanche, par des conditions météomarines très calmes.

L'analyse portant sur les profils montre des résultats moins tranchés. Si ce schéma semble à peu près fonctionner pour le cordon dunaire du Grand Rocher (fig. 10B), aucune relation allant clairement dans ce sens ne s'exprime pour le cordon dunaire de Saint-Michel-enGrève (fig. 10C). Cela s'explique par le fait que l'érosion induite par les phénomènes de run-up n'affecte pas l'ensemble du cordon dunaire mais uniquement certaines parties de ce dernier. Comme le montre la figure 12 , l'altitude du pied de dune conditionne l'importance des phénomènes érosifs dès lors qu'elle est inférieure à la hauteur du niveau d'eau extrême (marée observée + run-up). Sur l'ensemble de la période d'observation, ces hauts niveaux marins ont atteint le pied de dune du Grand Rocher bien plus fréquemment que celui de Saint-Michel-enGrève (fig. 12A et 12B). Comme on l'a vu précédemment, cela s'est traduit par un engraissement continu de ce dernier tout au long de la période d'observation. On note que les hauts niveaux marins correspondant à la marée observée stricto sensu ne jouent pas dans l'érosion des dunes, ce qu'illustre la figure 12. Dans la majorité des cas, cette variable structure le deuxième axe factoriel mais en tant qu'élément isolé. Cela montre que les niveaux de pleine mer de marée observée sont infé-

rieurs à l'altitude des pieds de dunes et que, comme l'a indiqué P.A. Pirazzoli (2000), les plus fortes marées de viveeau ont rarement coïncidé avec un épisode tempéteux de très forte intensité.

Enfin, comme on l'a vu précédemment, la saisonnalité s'exprime particulièrement bien au niveau des périodes 2 et 3 (fin du mois d'octobre au début du mois de décembre 2002) qui, dans tous les cas, s'opposent fortement aux périodes 10 et 11 (mi-mai au début du mois de septembre 2003) (fig. 13). Pour le reste des individus on note une forte dispersion qui brouille ce fonctionnement saisonnier. Cela peut s'expliquer par l'ensemble des processus agissant durant les périodes de régénération des cordons dunaires qui, comme on l'a dit précédemment, peuvent gommer l'ef- fet des tempêtes. L'estimation du transit éolien, couplé aux précipitations, illustre bien ces mécanismes (tab. 5). Durant les périodes 1 et 2 marquées par de fortes tempêtes, les valeurs théoriques des volumes sédimentaires transportés vers les cordons dunaires sont considérables. Toutefois, si l'on tient compte des précipitations importantes enregistrées durant ces périodes ( $\geq 1 \mathrm{~mm} /$ jour), on peut penser que les déplacements sédimentaires ont été quasiment nuls, en raison du rôle limitant du taux d'humidité dans le transport éolien (Svasek et Terwindt, 1974 ; Sarre, 1988 ; Arens, 1996b ; Meur-Ferrec et Ruz, 2002). De ce fait, la régénération des cordons dunaires durant ces périodes a été inexistante, d'où la forte signature des épisodes morphogènes sur la morphologie. À l'inverse, pour les autres 


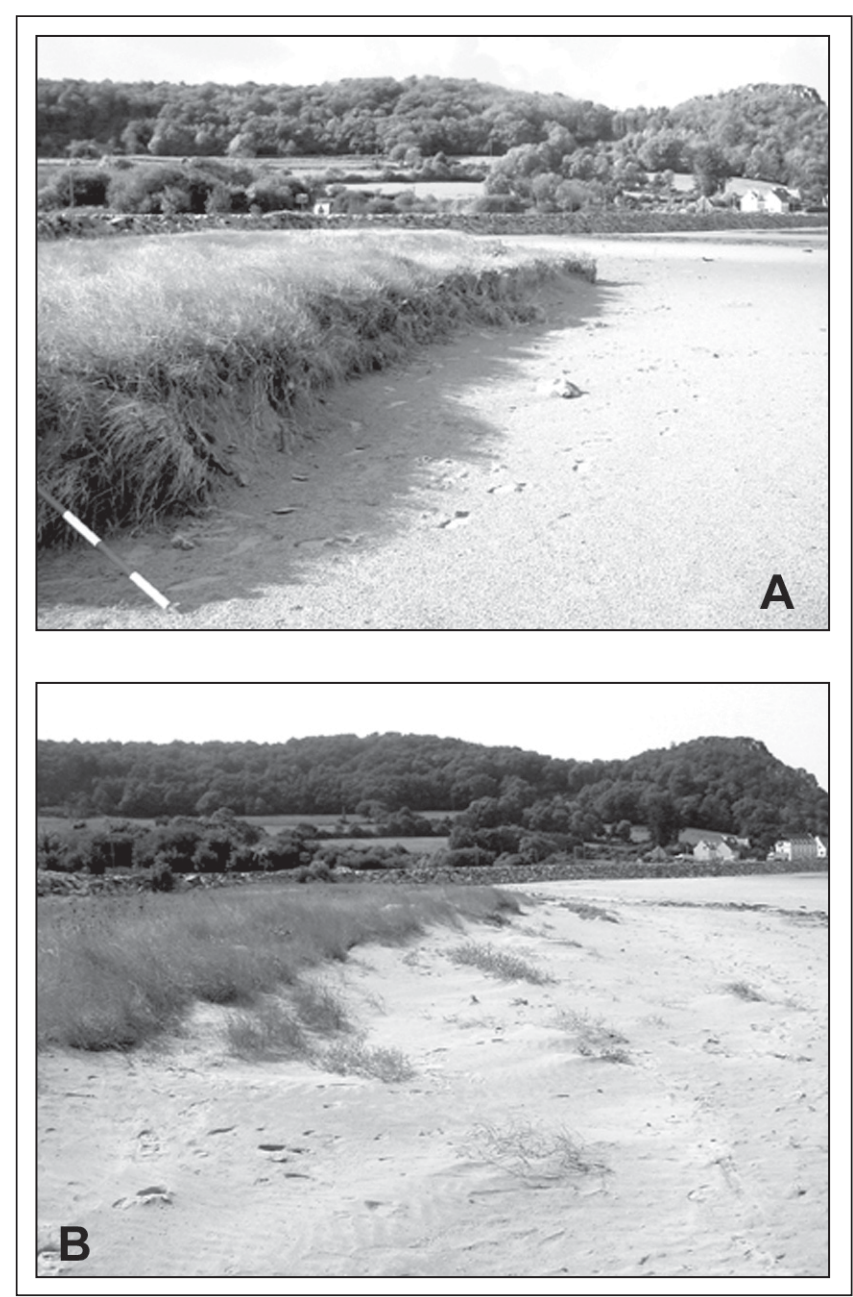

périodes hivernales et printanières (périodes 4 , 6, 7 et 8), l'impact morpho-sédimentaire des tempêtes a été en partie gommé par un engraissement éolien plus ou moins important favorisé par un temps sec.

\section{Discussion}

D'une façon générale, les résultats obtenus sont en accord avec le schéma classique du fonctionnement saisonnier des cordons dunaires décrit dans la littérature (Davidson-Arnott et Law, 1990 ; Sherman et Bauer, 1993 ; Carter et al., 1990 ; Hesp, 1981 ; Hallégouët, 1981). À la période automnale-hivernale de forte activité morphogène marquée par un stade érosif succède une phase de régénération, particulièrement efficace durant l'été. Toutefois, lorsque l'on compare le fonctionnement des deux secteurs étudiés, des différences apparaissent. Elles rendent compte de spécificités morphosédimentaires et hydrodynamiques propres à chacun des sites. Ces dernières permettent de replacer le fonctionnement de ces deux cordons dunaires au sein des modèles conceptuels (Short et Hesp, 1982 ; Psuty, 1992 ; Hesp, 2002 ; Ruz, 2004).
Fig. 13 - Exemples de morphologie du haut de plage et du front dunaire, dune du Grand Rocher. A : présence d'une falaise d'érosion (cliché : $23 / 10 / 2002$, S. Suanez). B : accumulation en pied de dune et végétation pionnière (cliché : 12/09/2003, S. Suanez).

Fig. 13 - Examples of the morphology of the upper beach and dune front, Grand Rocher dune. A: erosional dune scarp (photo: 23/10/2002, S. Suanez). B: sand accumulation on the dune toe and pioneer plants (photo: 12/09/2003, S. Suanez).

Si l'on se fonde sur le modèle de A.D. Short et P.A. Hesp (1982) reposant sur une classification morphodynamique des environnements côtiers, les cordons dunaires du Grand Rocher et de Saint-Michel-en-Grève devraient être très étendus et bénéficier d'une alimentation sédimentaire très importante. En suivant ce schéma, d'une part le caractère « hyper-dissipant » de la baie de Saint-Michel-en-Grève minimiserait les phénomènes érosifs par un fort amortissement de la houle, d'autre part, la grande largeur de l'estran très plan expliquerait un transport éolien de la plage vers la dune très important. Si pour le cordon dunaire de Saint-Michelen-Grève ce modèle peut s'appliquer, pour celui du Grand Rocher marqué par une forte érosion, le fonctionnement apparaît bien plus complexe.

Le modèle proposé par N.P. Psuty (1992) reposant sur la notion de bilan sédimentaire du couple plage / dune semble plus approprié (fig. 14). Ainsi, on peut classer le cordon dunaire de Saint-Michel-en-Grève dans le scénario (B) décrivant une situation où le bilan sédimentaire de la plage et de la dune est positif. Dans ce contexte, l'auteur souligne l'existence de conditions propices à l'édification de dunes bordières successives au fur et à mesure de la progression du rivage. Cette disposition rappelle à bien des égards la mor-

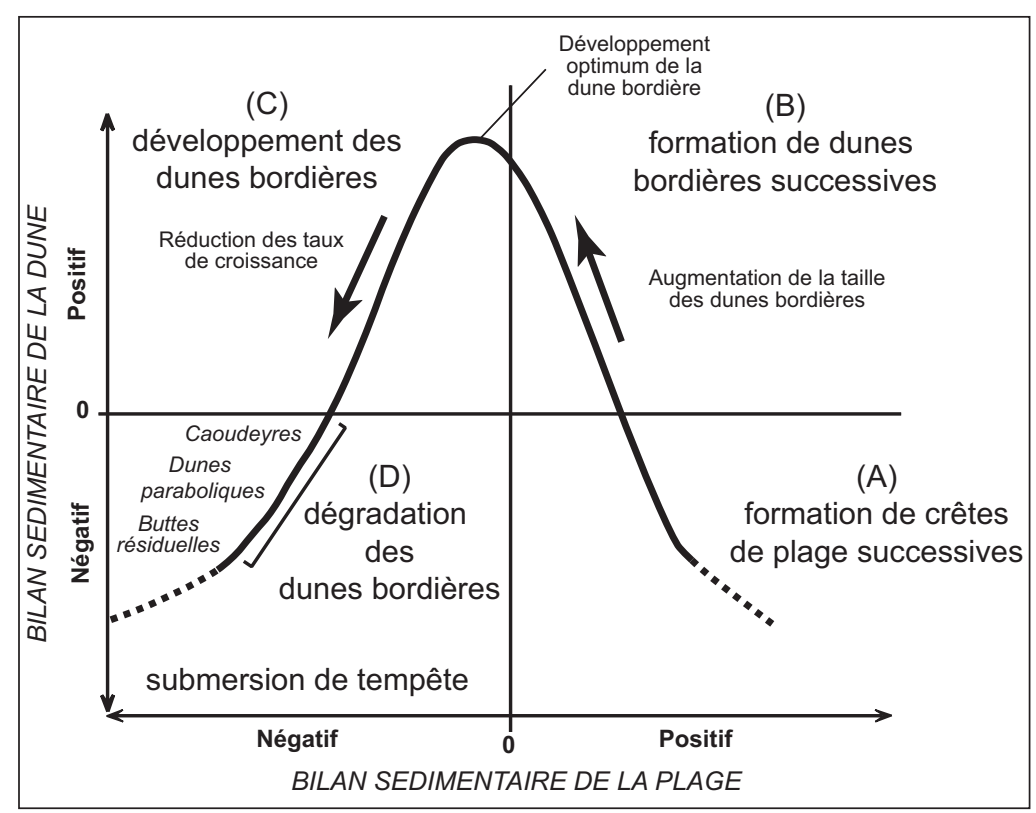

Fig. 14 - Modèle de formation et d'évolution des cordons dunaires fondé sur le bilan sédimentaire de la plage et de la dune (d'après N.P. Psuty, 1992).

Fig. 14 - Model of construction and evolution of coastal dunes based on beach and dune sediment budget (from Psuty, 1992). 
phologie décrite précédemment. À l'inverse, le cordon dunaire du Grand Rocher se situerait plutôt entre les scénarios $(D)$ et $(C)$ décrivant respectivement des situations où le bilan sédimentaire du couple plage / dune est déficitaire, déficitaire pour la plage et équilibré à positif pour la dune. Dans ce contexte on assiste à une dégradation du cordon dunaire liée à l'action des phénomènes de submersion (onde de tempête et/ou PM de vive-eau) avec, durant les périodes de faible activité morphogène, des courtes phases de régénération. Ces notions portant sur la relation entre le bilan sédimentaire et la dynamique des cordons dunaires ont en partie été analysées et décrites par S. Suanez (2004).

Enfin, le modèle proposé par P.A. Hesp (1988, 2002) reposant sur une classification morpho-écologique permet de faire la synthèse entre les dynamiques agissant à court terme et l'évolution à plus long terme des cordons dunaires. Ainsi, le cordon dunaire de Saint-Michel-en-Grève appartiendrait au stade 2 correspondant à une dune bordière bien constituée et bien végétalisée (70 à $90 \%$ de couverture végétale), pouvant être précédée de dunes embryonnaires. Dans ce contexte, des formes mineures d'érosion (sifflevent) peuvent affecter la morphologie du versant externe ; toutefois, sur le long terme, ce cordon évoluerait vers un stade 2A. Ce dernier caractérise les côtes stables ou en accumulation et se distingue par un développement de la dune par exhaussement et/ou formation de crêtes dunaires successives. Sur du court terme, on peut avoir un stade 2D décrivant une érosion en micro-falaise par les vagues de tempête. Néanmoins, pour le cordon dunaire de SaintMichel-en-Grève, la réversibilité de ces phénomènes s'illustre par un ensevelissement rapide de ces formes d'érosion et une revégétalisation du versant externe.

Le cordon dunaire du Grand Rocher reste difficilement classable tant les processus de recul qui l'affectent sont rapides. Ainsi, les cycles d'érosion décrits par l'auteur ont du mal à s'appliquer dans ce cas. Du point de vue morphoécologique et de la dynamique, il appartiendrait à un stade intermédiaire entre les stades $2 \mathrm{~B}$ et $3 \mathrm{~B}$, stade marqué par un recul continu sur le long terme. Sur le court terme, la dynamique serait liée à l'action incessante de l'érosion entaillant en micro-falaise le versant externe qui jamais ne retrouverait sa position initiale malgré des phases temporaires de réalimentation.

\section{Conclusion}

Le suivi à haute fréquence réalisé dans le cadre de cette étude a permis d'appréhender de façon satisfaisante les changements morphosédimentaires rapides des cordons dunaires. L'expérience montre que les deux méthodes de mesure mises en œuvre apparaissent complémentaires et utiles. Si la mesure le long des profils permet de cerner les modifications sur l'ensemble du système plage / dune, elle reste toutefois très dépendante de la localisation du profil. Cela introduit une part importante de subjectivité dans les résultats obtenus qui sont alors difficilement généralisables à l'ensemble du cordon dunaire. La mesure du front de dune permet d'éliminer cette incertitude et de donner des résultats plus pertinents à partir de l'évolution des surfaces dunaires. Toutefois, elle ne peut s'appliquer que pour des cordons dunaires présentant une morphologie assez simple et dont la limite frontale est facilement identifiable, ce qui est le cas des cordons dunaires de la baie de Saint-Michel-en-Grève.

Le recours à l'Analyse factorielle en Composante Principale $(\mathrm{ACP})$ apparaît efficace et relativement simple à mettre en œuvre. Elle a permis d'une part de montrer le rôle important que pourrait jouer les phénomènes de run-up associés aux fortes houles de tempêtes dans le recul du cordon dunaire du Grand Rocher, confirmant ainsi la grande fragilité de ce secteur. D'autre part, elle souligne le rôle limité des hauts niveaux de marée de vive-eau qui, dans la plupart des cas, n'atteignent jamais le pied de dune.

Enfin, l'analyse des forçages météo-marins et des dynamiques morphosédimentaires montre un fonctionnement saisonnier bien marqué entre l'hiver et l'été. Toutefois, les mécanismes associés aux courtes périodes de faible activité morphogène venant s'intercaler aux épisodes tempétueux brouillent quelque peu ce schéma. Comme l'ont montré plusieurs auteurs, cela souligne les interactions entre les processus agissant à différentes échelles temporelles (Davidson-Arnott, 1988 ; Thom et Hall, 1991 ; Battiau-Queney et al., 2000 ; Battiau-Queney, 2004), c'est-à-dire saisonnière et épisodique dans le cas présent.

\section{Remerciement}

Nous tenons à remercier le Conseil général des Côtes d'Armor qui a financé cette étude dans le cadre du Programme Prolittoral, volet curatif. Un grand merci à P.A. Meunier de Géomer pour son aide apportée au traitement des données, à J.-C. Fournier, M. Benoît et F. Lafon du LNHE - EDF pour la fourniture des données de houles, et à B. Simon du SHOM (Brest) pour la fourniture des données marégraphiques. Nous remercions également les relecteurs qui, par leurs remarques et leurs suggestions, nous ont permis d'améliorer le contenu de ce texte. Enfin, merci à A. Héquette pour son aide apportée aux dernières relectures et aux traductions en langue anglaise.

\section{Références}

Alcantaro-Carrio J., Alonso I. (2002) - Measurement and prediction of aeolian sediment transport at Jandra Isthmus (Fuerteventura, Canary Islands). Journal of Coastal Research, 18 (2), 300-315.

Arens S.M. (1994) - Aeolian Processes in the Dutch Foredunes. Thèse de doctorat, université d'Amsterdam (Eds.), Technical Advisory committee of Water defences, $150 \mathrm{p}$.

Arens S.M. (1996a) - Sediment dynamics of a coastal foredune at Schiermonnikoog, The Netherlands. In Jones P.S., Healy M.G., Williams A.T. (Eds.), Studies in European Coastal Management, Samara Publications, Cardigan, 137-146.

Arens S.M. (1996b) - Rates of aeolian transport on a beach in a temperate humid climate. Geomorphology, 17, 3-18.

Arens S.M. (1997) - Transport rates and volume changes in a coastal foredune on a Dutch Wadden island. Journal of Coastal Conservation, 3, 49-56. 
Audran E. (1999) - Régimes comparés des vents forts sur l'île de Groix et de Belle-Ile (Morbihan). Méditerranée, 95, 75-76.

Bagnold R.A. (1941) - The physics of blown sand and desert dunes. Chapman and Hall, London, 265 p.

Battiau-Queney Y. (2004) - Haut de plage et front dunaire : enregistrement morphologique de la dynamique éolienne et marine dans un système macrotidal. Bulletin de l'Association de Géographes Français, 3, 393-404.

Battiau-Queney Y., Fauchois J., Deboudt P., Lanoy-Ratel P. (2000) - Beach-dune systems in a macrotidal environment along the northem French coast (English Channel and southern North Sea). Journal of Coastal Research, Special Issue, 34, 580-592.

Battjes J.A. (1974) - Surf similarity. Proceeding of the 14th Coastal Engineering Conference, American Society of Civil Engineers, 466-479.

Betts N.L., Orford, J.D., White D., Graham C.J. (2004) - Storminess and surges in the South Western Approaches of the eastern North Atlantic: the synoptic climatology of recent extreme coastal storms. Marine Geology, 210, 227-246.

Bouligand R., Pirazzoli P.A. (1999) - Les surcotes et les décotes marines à Brest, étude statistique et évolution. Oceanologica Acta, 22-2, 153-166.

Bruzzi C. (1996) - Introduction méthodologique à l'étude des tempêtes. Exemple du littoral du delta du Rhône. Méditerranée, $4,51-54$

Bruzzi C. (1998) - Les tempêtes et l'évolution morphosédimentaire des plages orientales du delta du Rhône. Thèse de doctorat, université de Provence Aix-Marseille I, 325 p.

Carter R.G.W., Hesp P.A., Nordstrom K.F. (1990) - Erosional landforms. In Nordstrom K.F., Psuty N.P., Carter R.G.W. (Eds.), Coastal dunes, form and process, John Wiley, London, 217-249.

Chamussy H., Charre J., Dumolard P., Durand M.-G., Le Berre M. (1994) - Initiation aux pratiques statistiques en géographie. Groupe Chadule, Masson, 3e édition, 203 p.

Clabaut P., Chamley H., Marteel H. (2000) - Evolution récente des dunes littorales à l'est de Dunkerque (Nord de la France). Géomorphologie : relief, processus, environnement, 2, 125-137.

Dail, H.J., Merrifield M.A., Bevis M. (2000) - Step beach morphology changes due to energetic wave forcing. Marine Geology, 162, 443-458.

Davidson-Arnott R.G.D. (1988) - Temporal and spatial controls on beach - dune interaction, Long Point, Lake Erie. In Psuty N.P. (Eds.), Journal of Coastal Research, Special Issue (3), 131-136.

Davidson-Arnott R.G.D., Law M.N. (1990) - Seasonal patterns and controls on sediment supply to coastal foredunes, Long Point, Lake Erie. In Nordstrom K.F., Psuty N.P., Carter R.W.G. (Eds.), Coastal dunes, form and process, John Wiley, London, 177-200.

Fichaut B., Hallégouët B. (1989) - Banneg : une île dans la tempête. Penn ar Bed, 135, 36-43.

Gad L., Suanez S., Hénaff A., Lidou F., Morel V. (2003) Apport de la géomorphologie à une problématique environnementale : comment faciliter le ramassage des algues vertes (Ulva armoricana) en baie de Saint-Michel-en-Grève (Côtes-d'Armor) ? Les Cahiers Nantais, 59, 89-102.

Hallégouët B. (1981) - Les crêtes littorales dunifiées du Massif Armoricain : formation et évolution, Géographie physique et Quaternaire, 35, 205-218.
Hénaff A. (2004) - Les aménagements des littoraux de la Région Bretagne en vue de leur défense contre l'érosion depuis 1949. Bulletin de l'Association de Géographes Français, 3, 346-359.

Hesp P.A. (1981) - The formation of shadow dunes. Journal of Sedimentary Petrology, 51 (1), 101-112.

Hesp P.A. (1988) - Morphology, dynamics and internal stratification of some established foredunes in southeast Australia. Journal of Sedimentary Geology, 55, 17-41.

Hesp P.A. (2002) - Foredunes and blowouts: initiation, geomorphology and dynamics. Geomorphology, 48, 245-268.

Holman R.A. (1986) - Extreme value statistics for wave run-up on a natural beach. Coastal Engineering, 9, 527-544.

Kawamura R. (1964) - Study of sand movement by wind. Hydraulic Engineery Laboratory, Technical Report HEL-28, Berkeley University, California, 99-108.

Komar P.D. (1998) - Beach processes and sedimentation, 2e édition, Prentice Hall, 544 p.

Lemasson L., Régnauld H. (1997) - Évolution trentenaire des vents littoraux sur le Grand Ouest français. Norois, 175, 417-431.

Masselink G., Hughes M.G. (2003) - Coastal processes and geomorphology. Arnold, London, 354 p.

Meur-Ferrec C., Ruz M.-H. (2002) - Transports éoliens réels et théoriques en haut de plage et sommet de dune (Wissant, Pas-deCalais, France). Géomorphologie : relief, processus, environnement, 4, 321-334.

Nielsen P., Hanslow D.J. (1991) - Wave runup distributions on natural beaches. Journal of Coastal Research, 7 (4), 1139-1152.

Pirazzoli P.A. (2000) - Surges, atmospheric pressure and wind change and flooding probability on the Atlantic coast of France. Oceanologica Acta, 23-6, 643-661.

Pirazzoli P.A., Regnauld H., Lemasson L. (2004) - Changes in storminess and surges in western France during the last century. Marine Geology, 210, 307-323.

Psuty N.P. (1988) - Sediment budget and beach/dune interaction. Journal of Coastal Research, Special Issue (3), 1-4.

Psuty N.P. (1992) - Spatial variation in coastal foredune development. In Carter R.W.G., Curtis T.G.F., Sheehy-Skefington (Eds.), Coastal dunes, geomorphology, ecology and management for conservation, A.A. Balkema, Rotterdam, 3-13.

Pye K. (1991) - Beach deflation and backshore dune formation following erosion under storm surge conditions: an example from Northwest England. Acta Mechanica, 2, 171-181.

Pye K., Neal A. (1994) - Coastal dune erosion at Formby Point, north Merseyside, England: causes and mechanisms. Marine Geology, 119, 39-56.

Ruggiero P., Kaminsky G.M., Komar P.D., McDouglas W.G. (1997) - Extreme waves and coastal erosion in the Pacific Northwest. Ocean Wave Measurement and Analysis, Proceeding of the 3rd International Symposium, Waves '97, 947-961.

Ruggiero P., Komar P.D., McDouglas W.G., Marra J.J., Beach R.A. (2001) - Wave runup, extreme water levels and erosion of properties backing beaches. Journal of Coastal Research, 17 (2), 407-419.

Ruz M.-H. (2004) - Formation et évolution des dunes bordières : modèles conceptuels. Bulletin de l'Association de Géographes Français, 3, 383-392.

Ruz M.-H., Meur-Ferec C. (2004) - Influence of high water levels on aeolian sand transport: upper beach/dune evolution on 
a macrotidal coast, Wissant Bay, northern France. Geomorpho$\log y, 60,73-87$.

Sarre R. (1988) - Evaluation of aeolian sand transport equations using intertidal zone measurements, Saunton Sands, England. Sedimentology, 35, 671-679.

Sherman D.J., Bauer B.O. (1993) - Dynamics of beach-dune systems. Progress in Physical Geography, 17 (4), 413-447.

Sherman D.J., Nordstrom K.F. (1985) - Beach scarps. Zeitschrift für Geomorphologie, 29, 139-152.

Shih S.-M., Komar P.D. (1994) - Sediments, beach morphology and sea cliff erosion within an Oregon coast littoral cell. Journal of Coastal Research, 10, 144-157.

Short A.D., Hesp P.A. (1982) - Wave, beach and dune interactions in southeast Australia. Marine Geology, 48, 259-284.

Stéphan P., Suanez S. (2004) - Dynamique morphosédimentaire des cordons dunaires de Tréduder et de Saint-Michel-en-Grève (baie de Saint-Michel-en-Grève - Côtes d'Armor) entre 1990 et 2003. Bulletin de l'Association de Géographes Français, 3, 438-448.

Stéphan P., Suanez S. (2005) - Mobilité du trait de côte en baie de Saint-Michel-en-Grève (Côtes d'Armor) ou le résultat d'une plage en accrétion. In D. Levacher, P. Sergent, A. Ouahsine et le Centre Français du Littoral (Eds.), Actes du colloque des VIIIe Journées Nationales Génie Côtier - Génie Civil, Compiègne 79 septembre 2004, Tome 1, 357-364.
Stéphan P. (2003) - Dynamiques morphosédimentaires récente et actuelle des cordons dunaires de la baie de Saint-Michel-enGrève. Mémoire de maîtrise de Géographie, université de Bretagne Occidentale, 309 p.

Suanez S. (2004) - Modifications morphodynamiques et phénomènes rétroactifs en baie de Saint-Michel-en-Grève (Côtesd'Armor). Bulletin de l'Association de Géographes Français, 3, 334-345.

Suanez S., Gad L., Hénaff A., Lidou F. (2002) - Géomorphologie appliquée et algues vertes : l'exemple en baie de Saint-Michel-en-Grève. Penn ar Bed, 187, 1-13.

Suanez S., Dehouck A., Stéphan P., Fichaut B., Cuq V. (2005) - Estimation par traitement statistique des marges d'erreur inhérentes à la mesure tachéométrique en géomorphologie, Séminaire de l'UMR LETG 6554 CNRS : Instrumentation et mesures de terrain, 30 janvier 2004 à Rennes 2 (http://letg.univnantes.fr/SeminaireUMR30012004MP.pdf), 1-10.

Svasek J.N., Terwindt J.H.J. (1974) - Measurements of sand transport by wind on a natural beach. Sedimentology, 21, 311-322.

Thom B.G., Hall W. (1991) - Behaviour of beach profiles during accretion and erosion dominated periods. Earth Surface Processes and Landforms, 16, 113-127.

Article reçu le 23 novembre 2004, accepté le 12 juin 2006. 